\title{
Electromagnetic control of valley splitting in ideal and disordered Si quantum dots
}

\author{
Amin Hosseinkhani $\odot^{*}$ and Guido Burkard $\odot^{\dagger}$ \\ Department of Physics, University of Konstanz, D-78457 Konstanz, Germany
}

(Received 30 June 2020; revised 22 September 2020; accepted 7 October 2020; published 3 November 2020)

\begin{abstract}
In silicon spin qubits, the valley splitting must be tuned far away from the qubit Zeeman splitting to prevent fast qubit relaxation. In this work, we study in detail how the valley splitting depends on the electric and magnetic fields as well as the quantum dot geometry for both ideal and disordered $\mathrm{Si} / \mathrm{SiGe}$ interfaces. We theoretically model a realistic electrostatically defined quantum dot and find the exact ground and excited states for the out-ofplane electron motion. This enables us to find the electron envelope function and its dependence on the electric and magnetic fields. For a quantum dot with an ideal interface, the slight cyclotron motion of electrons driven by an in-plane magnetic field slightly increases the valley splitting. Importantly, our modeling makes it possible to analyze the effect of arbitrary configurations of interface disorders. In agreement with previous studies, we show that interface steps can significantly reduce the valley splitting. Interestingly, depending on where the interface steps are located, the magnetic field can increase or further suppress the valley splitting. Moreover, the valley splitting can scale linearly or, in the presence of interface steps, nonlinearly with the electric field.
\end{abstract}

DOI: 10.1103/PhysRevResearch.2.043180

\section{INTRODUCTION}

The spin of isolated electrons trapped in silicon-based heterostructures is very promising for building high performance and scalable qubits [1]. The long relaxation time [2-4] and dephasing time [5-7] that are achieved in these qubits are due to the weak spin-orbit interaction and nuclear zero-spin isotopes. Strong coherent coupling between $\mathrm{Si}$ spin qubits and photons using superconducting resonators has been realized $[8,9]$ while the fidelities demonstrated for single and two-qubit gates are steadily improving [10-14]. Having mentioned all these advantages, we note that the nature of the degenerate conduction band minima, known as valleys, in bulk silicon poses a significant challenge for the operation and scalability of silicon spin qubits. It can be shown that a combination of biaxial strain as well as the sharp interface potential lifts the valley degeneracy in Si heterostructures, and gives rise to two low-lying states [1]. In general, a qubit performs well only when the qubit energy splitting is well-separated from any other energy scale in the environment. In Si spin qubits, the spin couples to the valley degree of freedom due to interfaceinduced spin-orbit interaction [15-17]. If the valley splitting becomes equal to the qubit Zeeman splitting, a condition known as spin-valley hotspot, the valley-spin mixing for the qubit excited state reaches its maximum and gives rise to a very fast qubit relaxation via electron-phonon interaction

\footnotetext{
*amin.hosseinkhani@uni-konstanz.de

${ }^{\dagger}$ guido.burkard@uni-konstanz.de
}

Published by the American Physical Society under the terms of the Creative Commons Attribution 4.0 International license. Further distribution of this work must maintain attribution to the author(s) and the published article's title, journal citation, and DOI.
$[3,18]$. It is, therefore, of crucial importance to understand how the valley splitting behaves as a function of parameters of the system; namely, electric and magnetic fields, the quantum dot geometry and the roughness at the $\mathrm{Si} /$ barrier interface.

In studying the valley splitting, a suitable starting point is the effective mass theory that can be used to obtain the electron envelope function. This envelope function, in turn, depends on the above-mentioned system parameters, and the valley splitting can be deduced from it. As we review in Sec. II A, in the absence of a magnetic field, the Hamiltonian describing the full envelope function is separable. While the in-plane envelope function is trivially given by the harmonic-oscillator wave function (due to the in-plane parabolic confinement), to our knowledge, the (ground state of the) out-of-plane envelope function has only been studied and approximated via variational methods [19-22] or by setting the barrier potential to infinity [23]. However, the assumptions involved in these methods render them less accurate for higher electric fields. In this paper, we model a realistic potential profile for a $\mathrm{SiGe} / \mathrm{Si} / \mathrm{SiGe}$ quantum dot by taking into account both $\mathrm{Si} / \mathrm{SiGe}$ interfaces as well as an interface between $\mathrm{SiGe}$ and the insulating layer hosting the gate electrodes. Within this model, we then find the exact solution for the ground state as well as excited state envelope functions for the out-ofplane electron motion. We also extend our analysis to include $\mathrm{Si} / \mathrm{SiO}_{2}$ heterostructures in Appendix A.

In the presence of an in-plane magnetic field, a cyclotron motion of electrons takes place which tends to increase the electron probability amplitude at the $\mathrm{Si} / \mathrm{SiGe}$ interface [24]. This effect can, in turn, modify and increase the valley splitting. The magnetic field couples in-plane to out-of-plane degrees of freedom and thus prevents us from finding the exact solution for the electron envelope function. Using the exact excited states for the out-of-plane envelope function, we find the full envelope function in the presence of a magnetic field 
by applying perturbation theory. We show that an in-plane magnetic field indeed slightly increases the valley splitting; up to a few Tesla, the valley splitting increases quadratically with the magnetic field. Besides this, for a quantum dot with an ideal interface, i.e., no miscuts and steps at the interface, we find that the dominant contribution to the valley splitting scales linearly with the electric field, $F_{z}$.

During the experimental process of fabricating silicon heterostructures, the formation of steps and miscuts at the $\mathrm{Si} / \mathrm{SiGe}$ seems to be inevitable $[25,26]$. It has been shown that the presence of interface steps can severely suppress the valley splitting $[22,27,28]$. Here we again use the exact excited states for the out-of-plane envelope function in order to perturbatively treat the effects of interface steps to the envelope function. We argue that our modeling is applicable to any configuration for the interface disorder. We first study how the interface steps suppress the valley splitting in the absence of a magnetic field. We show that the valley splitting of a disordered quantum dot can scale either sublinearly, linearly or superlinearly with the electric field, depending on the step configuration. We then consider the effects of an in-plane magnetic field. While it has been speculated that the magnetic field can increase the valley splitting in the presence of interface steps [27,28], interestingly, we find that the magnetic field can both increase or further suppress the valley splitting depending on the locations of the steps.

This paper is structured as follows. In Sec. II A, we present our model and find the exact solution for the out-of-plane electron motion for a $\mathrm{SiGe} / \mathrm{Si} / \mathrm{SiGe}$ quantum dot. In Sec. II B, we obtain the envelope function in the presence of an in-plane magnetic field for a quantum dot with an ideal $\mathrm{Si} / \mathrm{SiGe}$ interface. In Sec. II C, we extend our model to include the interface disorders and derive the envelope function for a certain configuration of steps. In Sec. III, we build on our findings for the envelope function to obtain and discuss the valley splitting; in Secs. III A and III B, we study how the valley splitting of an ideal quantum dot depends on the electric and magnetic field field. In Sec. III C, we consider interface disorder and calculate the valley splitting and its phase depending on the location of the step. We then investigate the role of the electric and magnetic fields in modifying the valley splitting. In Sec. IV, we summarize and conclude the paper. Appendices contain further details of our analysis as well as some extensions of the theory to include an analysis of the out-of-plane envelope function and the valley splitting of $\mathrm{Si} / \mathrm{SiO}_{2}$ heterostructures.

\section{MODEL}

We consider a $\mathrm{SiGe} / \mathrm{Si} / \mathrm{SiGe}$ heterostructure grown along the $\hat{z}$ direction ([001]) while the silicon layer is between $-d_{t} \leqslant z \leqslant 0$. Figure 1(a) shows a schematic cross-section of the layer structure of the system. An electric field is applied along $\hat{z}$ via the gates, and we consider both interfaces between $\mathrm{Si}$ and $\mathrm{SiGe}$ at $z=0$ as well as $z=-d_{t}$. The energy offset between the minima of the conduction band in $\mathrm{Si}$ and $\mathrm{SiGe}$ is given by $U_{0}=150 \mathrm{meV}$. Moreover, we also consider the interface between the top $\mathrm{SiGe}$ barrier and the insulating layer that hosts the electric gates. Inside the insulating layer, we take $U_{\infty}=\infty$ which indicates the envelope function does not leak into that region. Panel (b) of Figure 1 shows the (a)

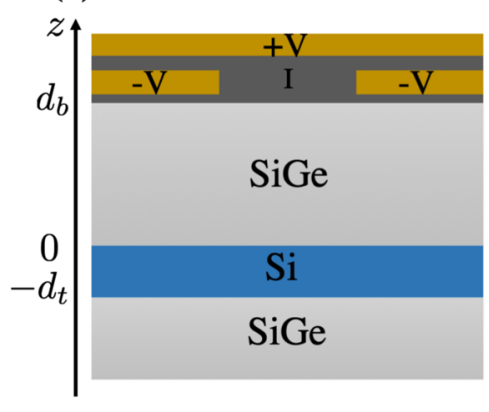

(b)

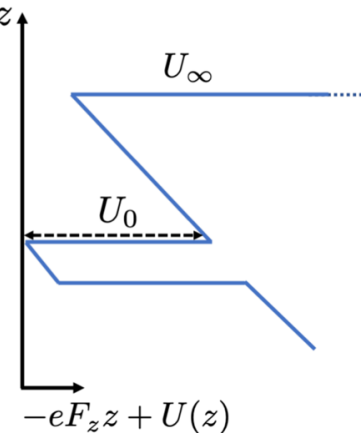

FIG. 1. (a) Schematic layered structure of a single $\mathrm{SiGe} / \mathrm{Si} / \mathrm{SiGe}$ quantum dot. The dark gray area (I) is an insulating layer hosting the electric gates. The $\pm V$ gates are used to trap and confine a single electron in the silicon layer. (b) The electrostatic potential profile along the growth direction $\hat{z} . F_{z}$ is the out-of-plane electric field generated by the gates, $U_{0}$ is the potential barrier of the SiGe layers, $U_{\infty}$ is the infinite potential barrier due to the insulating layer. The potential energy $U(z)$ is defined in Eq. (2).

full potential along $\hat{z}$. Moreover, here we assume a generally elliptical quantum dot with harmonic in-plane confinement. We denote the radius of the quantum dot along $\hat{x}$ by $x_{0}$ and the radius along $\hat{y}$ by $y_{0}$.

\section{A. Exact envelope function in absence of a magnetic field}

Within the effective mass theory and in the absence of a magnetic field, the Hamiltonian describing the envelope function reads

$$
\begin{aligned}
H_{x y z}= & \frac{p_{x}^{2}}{2 m_{t}}+\frac{1}{2} m_{t} \omega_{x}^{2} x^{2}+\frac{p_{y}^{2}}{2 m_{t}}+\frac{1}{2} m_{t} \omega_{y}^{2} y^{2} \\
& +\frac{p_{z}^{2}}{2 m_{l}}-e F_{z} z+U(z) .
\end{aligned}
$$

Here $m_{t}=0.19 m_{e}$ and $m_{l}=0.98 m_{e}$ are the transverse and longitudinal effective mass, $\omega_{x}=2 \hbar / m_{t} x_{0}^{2}$ and $\omega_{y}=2 \hbar / m_{t} y_{0}^{2}$ are the confinement frequencies along $\hat{x}$ and $\hat{y}$, and

$$
U(z)=U_{0} \theta\left(-z-d_{t}\right)+U_{0} \theta(z)+U_{\infty} \theta\left(z-d_{b}\right),
$$

where $d_{t}$ is the thickness of the Silicon layer and $d_{b}$ is the thickness of the upper SiGe barrier.

Eq. (1) clearly gives rise to a separable envelope function $\psi_{x y z}=\psi_{x} \psi_{y} \psi_{z}$ where $\psi_{x}$ and $\psi_{y}$ are the well-known harmonic oscillator wave functions. Our main objective in this section is to find the exact eigenstates $\psi_{z, n}$ and eigenenergies $E_{z, n}$ for the out-of-plane electron motion.

Given Eq. (1), we write the Schrödinger equation for the envelope function $\psi_{z, n}$ as

$$
\left\{\frac{p_{z}^{2}}{2 m_{l}}-e F_{z} z+U(z)\right\} \psi_{z, n}=E_{z, n} \psi_{z, n} .
$$

We now use the electrical confinement length,

$$
z_{0}=\left[\frac{\hbar^{2}}{2 m_{l} e F_{z}}\right]^{1 / 3}
$$


and its associated energy scale,

$$
\epsilon_{0}=\frac{\hbar^{2}}{2 m_{l} z_{0}^{2}}
$$

in order to piecewise expressing Eq. (3) as

$$
\begin{aligned}
& \frac{d^{2}}{d \tilde{z}^{2}} \psi_{z, n}-\left(\tilde{U}_{0}-\tilde{z}-\tilde{\epsilon}_{z, n}\right) \psi_{z, n}=0,0<z<d_{b}, \\
& \frac{d^{2}}{d \tilde{z}^{2}} \psi_{z, n}-\left(-\tilde{z}-\tilde{\epsilon}_{z, n}\right) \psi_{z, n}=0, \quad-d_{t} \leqslant z \leqslant 0, \\
& \frac{d^{2}}{d \tilde{z}^{2}} \psi_{z, n}-\left(\tilde{U}_{0}-\tilde{z}-\tilde{\epsilon}_{z, n}\right) \psi_{z, n}=0, z<-d_{t} .
\end{aligned}
$$

Here $\tilde{z}=z / z_{0}, \tilde{\epsilon}_{z, n}=E_{z, n} / \epsilon_{0}$, and $\tilde{U}_{0}=U_{0} / \epsilon_{0}$ are the normalized length, eigenenergy, and potential.

The above equation, at each interval, has generally two linearly independent solutions known as Airy functions of the first and second kind, $\mathrm{Ai}$ and $\mathrm{Bi}$ [29]. We thus find the exact solution for $\psi_{z, n}$ :

$$
\psi_{z, n}=N_{0} z_{0}^{-1 / 2} \begin{cases}c_{1} \operatorname{Ai}\left(\tilde{\zeta}_{n}\right)+c_{2} \operatorname{Bi}\left(\tilde{\zeta}_{n}\right), & 0<z<d_{b} \\ c_{3} \operatorname{Ai}\left(\tilde{\zeta}_{n}\right)+c_{4} \operatorname{Bi}\left(\tilde{\zeta}_{n}\right), & -d_{t} \leqslant z \leqslant 0 \\ c_{5} \operatorname{Ai}\left(\tilde{\zeta}_{n}\right), & z<-d_{t}\end{cases}
$$

where we defined

$$
\tilde{\zeta}_{n}= \begin{cases}\tilde{U}_{0}-\tilde{z}-\tilde{\epsilon}_{z, n}, & 0<z<d_{b} \\ -\tilde{z}-\tilde{\epsilon}_{z, n}, & -d_{t} \leqslant z \leqslant 0 . \\ \tilde{U}_{0}-\tilde{z}-\tilde{\epsilon}_{z, n}, & z<-d_{t}\end{cases}
$$

Note that the Bi function is omitted from the solution for $z<$ $-d_{t}$. This is based on the physical ground that $\mathrm{Bi}$ does not give rise to a decaying behavior inside the extended barrier layer.

In order to find the eigenenergies and determine the coefficients involved in the envelope function Eq. (7), we apply the appropriate boundary conditions; i.e., $\psi_{z, n}$ and its first derivative $\psi_{z, n}^{\prime}$ must be continuous at the interfaces between $\mathrm{Si}$ and $\mathrm{SiGe}$, at $z=0$ and $z=-d_{t}$. Moreover, since there is no leakage to the insulating layer, the envelope function must vanish at $z=d_{b}$. By imposing this set of boundary conditions, we obtain the equation below from which we can numerically find all possible eigenenergies,

$$
\begin{aligned}
f_{1}\left(\tilde{\epsilon}_{z, n}, \tilde{d}_{t}, \tilde{U}_{0}\right) & g_{1}\left(\tilde{\epsilon}_{z, n}, \tilde{d}_{b}, \tilde{U}_{0}\right) \\
& -f_{2}\left(\tilde{\epsilon}_{z, n}, \tilde{d}_{t}, \tilde{U}_{0}\right) g_{2}\left(\tilde{\epsilon}_{z, n}, \tilde{d}_{b}, \tilde{U}_{0}\right)=0,
\end{aligned}
$$

with the definitions

$$
\begin{aligned}
f_{1}\left(\tilde{\epsilon}_{z, n}, \tilde{d}_{t}, \tilde{U}_{0}\right)= & \operatorname{Bi}^{\prime}\left(\tilde{d}_{t}-\tilde{\epsilon}_{z, n}\right) \operatorname{Ai}\left(\tilde{U}_{0}+\tilde{d}_{t}-\tilde{\epsilon}_{z, n}\right) \\
& +\operatorname{Bi}\left(\tilde{d}_{t}-\tilde{\epsilon}_{z, n}\right) \operatorname{Ai}^{\prime}\left(\tilde{U}_{0}+\tilde{d}_{t}-\tilde{\epsilon}_{z, n}\right), \\
f_{2}\left(\tilde{\epsilon}_{z, n}, \tilde{d}_{t}, \tilde{U}_{0}\right)= & \operatorname{Ai}\left(\tilde{d}_{t}-\tilde{\epsilon}_{z, n}\right) \operatorname{Ai}^{\prime}\left(\tilde{U}_{0}+\tilde{d}_{t}-\tilde{\epsilon}_{z, n}\right) \\
& +\operatorname{Ai}^{\prime}\left(\tilde{d}_{t}-\tilde{\epsilon}_{z, n}\right) \operatorname{Ai}\left(\tilde{U}_{0}+\tilde{d}_{t}-\tilde{\epsilon}_{z, n}\right),
\end{aligned}
$$

and

$$
\begin{aligned}
& g_{1}\left(\tilde{\epsilon}_{z, n}, \tilde{d}_{b}, \tilde{U}_{0}\right) \\
& =\operatorname{Ai}\left(-\tilde{\epsilon}_{z, n}\right)\left[\operatorname{Bi}^{\prime}\left(\tilde{U}_{0}-\tilde{\epsilon}_{z, n}\right)-\operatorname{Ai}^{\prime}\left(\tilde{U}_{0}-\tilde{\epsilon}_{z, n}\right) \frac{\operatorname{Bi}\left(\chi_{n, d_{b}}\right)}{\operatorname{Ai}\left(\chi_{n, d_{b}}\right)}\right]
\end{aligned}
$$

$$
\begin{aligned}
& -\operatorname{Ai}^{\prime}\left(-\tilde{\epsilon}_{z, n}\right)\left[\operatorname{Bi}\left(\tilde{U}_{0}-\tilde{\epsilon}_{z, n}\right)-\operatorname{Ai}\left(\tilde{U}_{0}-\tilde{\epsilon}_{z, n}\right) \frac{\operatorname{Bi}\left(\chi_{n, d_{b}}\right)}{\operatorname{Ai}\left(\chi_{n, d_{b}}\right)}\right], \\
& g_{2}\left(\tilde{\epsilon}_{z, n}, \tilde{d}_{b}, \tilde{U}_{0}\right) \\
& =\operatorname{Bi}^{\prime}\left(-\tilde{\epsilon}_{z, n}\right)\left[\operatorname{Bi}\left(\tilde{U}_{0}-\tilde{\epsilon}_{z, n}\right)-\operatorname{Ai}\left(\tilde{U}_{0}-\tilde{\epsilon}_{z, n}\right) \frac{\operatorname{Bi}\left(\chi_{n, d_{b}}\right)}{\operatorname{Ai}\left(\chi_{n, d_{b}}\right)}\right] \\
& -\operatorname{Bi}\left(-\tilde{\epsilon}_{z, n}\right)\left[\operatorname{Bi}^{\prime}\left(\tilde{U}_{0}-\tilde{\epsilon}_{z, n}\right)-\operatorname{Ai}^{\prime}\left(\tilde{U}_{0}-\tilde{\epsilon}_{z, n}\right) \frac{\operatorname{Bi}\left(\chi_{n, d_{b}}\right)}{\operatorname{Ai}\left(\chi_{n, d_{b}}\right)}\right],
\end{aligned}
$$

where $\chi_{n, d_{b}}=\tilde{U}_{0}-\tilde{d}_{b}-\tilde{\epsilon}_{z, n}$ and $\mathrm{Ai}^{\prime}$ and $\mathrm{Bi}^{\prime}$ are the first derivatives of the $\mathrm{Ai}$ and $\mathrm{Bi}$ functions.

Once the (normalized) eigenenergy $\tilde{\epsilon}_{z, n}$ is found, we use it to calculate the coefficients $c_{1}$ to $c_{5}$. The coefficient $N_{0}$ is found by using the normalization of the envelope function. We note that by solving Eq. (9), we also find a set of states where the envelope function is not localized in the Si quantum well but rather in the upper SiGe barrier underneath the insulating layer. As we discuss it in Sec. III, the valley splitting is basically determined by the ground state localized in the $\mathrm{Si}$ quantum well. In the presence of a magnetic field or interface steps, we also need to take into account the excited states which have sizable overlap with the localized ground state in the Si quantum well; see Eqs. (28), (29), and (36). As such, the states that are localized underneath the insulating layer do not contribute to the behavior of the valley splitting, and we neglect them in this paper.

For the ground state of the electron motion along $\hat{z}$, we can simplify the analysis presented above and find analytic relations. As we show in Appendix A, the (normalized) ground-state energy in the regime of a deep quantum well, $\tilde{U}_{0} \gg 1$, can be expressed up to the leading order as

$$
\tilde{\epsilon}_{z, 0}=r_{0}-\tilde{U}_{0}^{-1 / 2}+\mathcal{O}\left(\tilde{U}_{0}^{-3 / 2}\right),
$$

where $-r_{0} \simeq-2.338$ is the smallest root (in absolute value) of the Ai function. The normalized envelope function in this case is approximated by,

$$
\psi_{z, 0}(\tilde{z}) \simeq \frac{z_{0}^{-1 / 2}}{\operatorname{Ai}^{\prime}\left(-r_{0}\right)} \begin{cases}\operatorname{Ai}\left(-\tilde{\epsilon}_{z, 0}\right) e^{-\frac{\mathrm{Ai}^{\prime}\left(-\tilde{\epsilon}_{z, 0}\right)}{\mathrm{Ai}\left(-\tilde{\epsilon}_{z, 0}\right)} \tilde{z}}, & \tilde{z}>0 \\ \operatorname{Ai}\left(-\tilde{z}-\tilde{\epsilon}_{z, 0}\right) . & \tilde{z} \leqslant 0\end{cases}
$$

In Fig. 2, we show the obtained energies for the ground state as well as first few excited states as a function of the applied electric field. In Fig. 3, we also show the probability density $\left|\psi_{z, n}\right|^{2}$ for the ground state and first two excited states for two different electric fields. In both figures, a comparison between the numerics and the analytical relations Eqs. (14) and (15) for the ground state shows a very good agreement.

We further note here that the interface-induced spin-orbit interaction is neglected in our model. Consideration of this effect has been shown to be essential for explaining the valley-dependent $g$ factor in silicon quantum dots $[16,17,23]$. However, as noted in Ref. [23], the matrix elements involved in the spin-orbit interaction are much smaller than the valley splitting matrix element. This justifies our omission of the interface-induced spin-orbit interaction. As we will show in the next sections, the information stored in the excited states $\psi_{z, n \geqslant 1}$ enables us to obtain the full envelope function $\psi_{x y z, 0}$ 


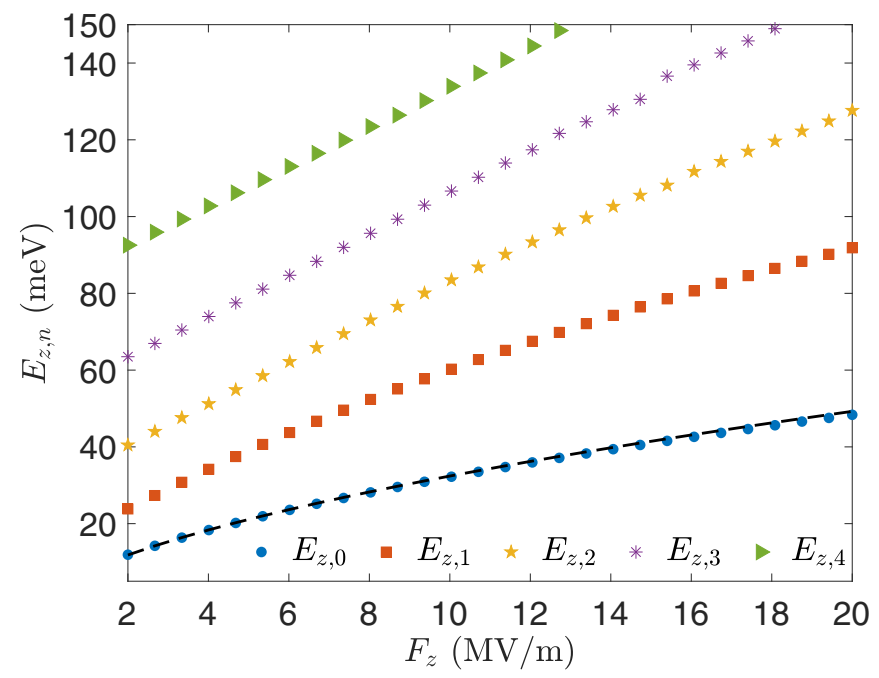

FIG. 2. Eigenenergies of the ground state $E_{z, 0}$ and first few excited states up to the $4^{\prime}$ th excited state $E_{z, 4}$ as a function electric field $F_{z}$. The symbols are obtained from numerically solving Eq. (9). The dashed line is the ground-state energy obtained from Eq. (14). The used quantum dot parameters are $d_{t}=10 \mathrm{~nm}$ and $d_{b}=46 \mathrm{~nm}$.

in a finite magnetic field, and also makes it possible to study realistic cases where there are steps and miscuts at the $\mathrm{Si} / \mathrm{SiGe}$ interface.

As we mention in Appendix A, in obtaining the simplified relations for the ground-state energy $\tilde{\epsilon}_{z, 0}$ and envelope function $\psi_{z, 0}$ given by Eqs. (14) and (15), we assume that due to the electric confinement, the envelope function of the

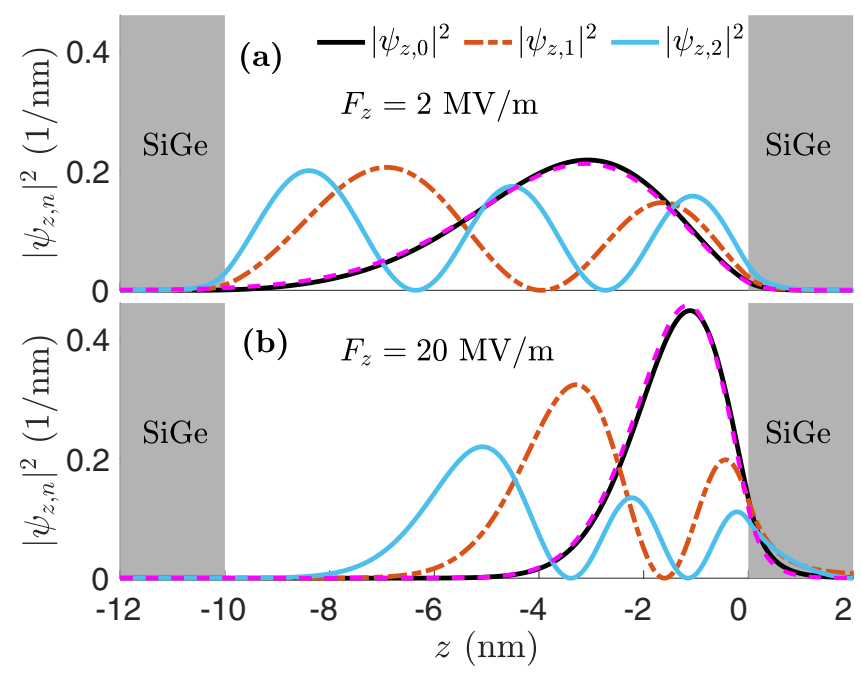

FIG. 3. Probability density of the ground state and the first and second excited states along $\hat{z}$. The gray areas mark the SiGe barriers. The solid and dash-dotted lines are found by using the exact envelope functions obtained from Eq. (7), while the eigeneregies are numerically found from Eq. (9); see Fig. 2. The dashed lines show the approximate ground-state envelope function given by Eq. (15). By comparing (a) with (b) we see that the envelope functions are pushed upwards by increasing the electric field $F_{z}$, as expected. The parameters for the quantum dot are the same as noted in the caption of Fig. 2. ground state has a negligible amplitude at the lower $\mathrm{SiGe} / \mathrm{Si}$ interface. Therefore the ground state becomes independent on the silicon layer thickness, $d_{t}$. In order to quantify the regime at which this assumption is valid, we note that, as far as the valley splitting is concerned, dropping the lower interface is justified provided $\left|\psi_{z, 0}\left(z=-d_{t}\right)\right|^{2} \ll \mid \psi_{z, 0}(z=$ $0)\left.\right|^{2}$. Taking $d_{t}=10 \mathrm{~nm}$, we find $F_{z} \gtrsim 2 \mathrm{MV} / \mathrm{m}$ validates the assumption of dropping the lower interface for the ground state. On the other hand, since the excited states have higher energies, they further spread within the silicon quantum well and, depending on the electric field, they can have sizable amplitude at the lower interface; see Fig. 3(a). Therefore, to properly determine the excited states, we always consider the lower $\mathrm{SiGe} / \mathrm{Si}$ interface by numerically solving Eq. (9). The relevant electric field for the $\mathrm{SiGe} / \mathrm{Si} / \mathrm{SiGe}$ heterostructures is typically cited in the literature to be $F_{z}=15 \mathrm{MV} / \mathrm{m}$ [21,30]. At this value for the electric field, the ground-state envelope function, even in the presence of magnetic field and interface steps, is found to have negligible amplitude at the lower interface so long as $d_{t} \gtrsim 5 \mathrm{~nm}$; see Figs. 4 and 6 (the silicon layer thickness is reported to be $d_{t}=12 \mathrm{~nm}$ in the experiment in Ref. [26] whereas $d_{t}=8 \mathrm{~nm}$ in the experiment in Ref. [31].) In Appendix A, we also consider a $\mathrm{Si} / \mathrm{SiO}_{2}$ heterostructure and find generalizations of Eqs. (14) and (15) to include analytical expressions not only for the ground state but also for the excited states as well.

\section{B. Envelope function in the presence of an in-plane magnetic field with ideal $\mathrm{Si} / \mathrm{SiGe}$ interface}

Let us now consider a quantum dot with an ideally flat $\mathrm{Si} / \mathrm{SiGe}$ interface in the presence of an in-plane magnetic field $\mathbf{B}=\left(B_{x}, B_{y}, 0\right)$. We use a gauge for which the vector potential becomes $\mathbf{A}=\left(0,0, y B_{x}-x B_{y}\right)$. By substituting $p_{z} \rightarrow p_{z}-e A_{z}(B)$ in Eq. (1), we arrive at the following form for the Hamiltonian describing the envelope function:

$$
H=H_{0}^{\prime}(\mathbf{B})+H_{\|}(\mathbf{B}) .
$$

where we start from the separable Hamiltonian,

$$
\begin{aligned}
H_{0}^{\prime}(\mathbf{B})= & \frac{p_{x}^{2}}{2 m_{t}}+\frac{1}{2} m_{t} \omega_{x}^{2}\left(B_{y}\right) x^{2}+\frac{p_{y}^{2}}{2 m_{t}}+\frac{1}{2} m_{t} \omega_{y}^{\prime 2}\left(B_{x}\right) y^{2} \\
& +\frac{p_{z}^{2}}{2 m_{l}}-e F_{z} z+U(z),
\end{aligned}
$$

and treat the couplings induced by the in-plane field as a perturbation,

$$
H_{\|}(\mathbf{B})=-B_{x} \frac{e}{m_{l}} y p_{z}+B_{y} \frac{e}{m_{l}} x p_{z}-B_{x} B_{y} \frac{e^{2}}{m_{l}} x y .
$$

We note that the confinement frequencies and lengths along $\hat{x}$ and $\hat{y}$ are modified by the magnetic field. Let us first define the cyclotron frequency and magnetic length induced by $B_{x(y)}$ by

$$
\begin{aligned}
& \Omega_{x(y)}=\frac{e B_{x(y)}}{\sqrt{m_{t} m_{l}}}, \\
& l_{x(y)}=\sqrt{\frac{\hbar}{e B_{x(y)}}} .
\end{aligned}
$$


TABLE I. The coefficients $\alpha_{n}$ and $\beta_{n}$ (in units of inverse Tesla). Here we used $F_{z}=15 \mathrm{MV} / \mathrm{m}$ (corresponding to $z_{0}=1.40 \mathrm{~nm}$ ), $x_{0}=$ $12 \mathrm{~nm}, y_{0}=15 \mathrm{~nm}$, and $B_{x}=B_{y}=5 \mathrm{~T}$.

\begin{tabular}{lcc}
\hline \hline$n$ & $\alpha_{n}\left(10^{-4} \mathrm{~T}^{-1}\right)$ & $\beta_{n}\left(10^{-4} \mathrm{~T}^{-1}\right)$ \\
\hline 1 & -9.18 & -8.76 \\
2 & 2.85 & 2.78 \\
3 & -1.38 & -1.35 \\
\hline \hline
\end{tabular}

We can then write

$$
\begin{gathered}
\omega_{x}^{\prime}=\omega_{x}\left(1+\frac{\Omega_{y}^{2}}{\omega_{x}^{2}}\right)^{1 / 2}, \\
\omega_{y}^{\prime}=\omega_{y}\left(1+\frac{\Omega_{x}^{2}}{\omega_{y}^{2}}\right)^{1 / 2}, \\
x_{0}^{\prime}=x_{0}\left(1+\frac{1}{4} \frac{m_{t}}{m_{l}} \frac{x_{0}^{4}}{l_{y}^{4}}\right)^{-1 / 4}, \\
y_{0}^{\prime}=y_{0}\left(1+\frac{1}{4} \frac{m_{t}}{m_{l}} \frac{y_{0}^{4}}{l_{x}^{4}}\right)^{-1 / 4} .
\end{gathered}
$$

In order to obtain the envelope function from Eq. (16), we treat $H_{0}^{\prime}(\mathbf{B})$ exactly and apply perturbation theory in $H_{\|}(\mathbf{B})$. The ground state up to the first-order perturbation then reads

$$
\Psi_{x y z, 0}(\mathbf{B})=\psi_{x y z, 0}^{(\mathbf{0})}(\mathbf{B})+\psi_{x y z}^{\|}(\mathbf{B}),
$$

where

$$
\psi_{x y z, 0}^{(\mathbf{0})}(\mathbf{B})=\psi_{x, 0}\left(B_{y}\right) \psi_{y, 0}\left(B_{x}\right) \psi_{z, 0}
$$

and

$$
\begin{aligned}
\psi_{x y z}^{\|}(\mathbf{B})= & -i B_{x} \frac{y_{0}^{\prime}}{z_{0}} \psi_{x, 0}\left(B_{y}\right) \psi_{y, 1}\left(B_{x}\right) \sum_{n=1}^{n_{\max }} \alpha_{n} \psi_{z, n} \\
& +i B_{y} \frac{x_{0}^{\prime}}{z_{0}} \psi_{x, 1}\left(B_{y}\right) \psi_{y, 0}\left(B_{x}\right) \sum_{n=1}^{n_{\max }} \beta_{n} \psi_{z, n} \\
& -B_{x} B_{y} x_{0}^{\prime} y_{0}^{\prime} \eta \psi_{x, 1}\left(B_{y}\right) \psi_{y, 1}\left(B_{x}\right) \psi_{z, 0},
\end{aligned}
$$

where the number of relevant bound excited states in the vertical direction for $F_{z}=15 \mathrm{MV} / \mathrm{m}$ is found to be $n_{\max }=3$, see Fig. 2. Here we defined the coefficients

$$
\begin{gathered}
\alpha_{n}=-\frac{1}{2} \hbar \frac{e}{m_{l}} \frac{\left\langle\psi_{z, 0}|\partial / \partial \tilde{z}| \psi_{z, n}\right\rangle}{E_{z, 0}-E_{z, n}-\hbar \omega_{y}^{\prime}} \\
\beta_{n}=-\frac{1}{2} \hbar \frac{e}{m_{l}} \frac{\left\langle\psi_{z, 0}|\partial / \partial \tilde{z}| \psi_{z, n}\right\rangle}{E_{z, 0}-E_{z, n}-\hbar \omega_{x}^{\prime}} \\
\eta=-\frac{1}{4} \frac{e^{2}}{m_{l}} \frac{1}{\hbar \omega_{x}^{\prime}+\hbar \omega_{y}^{\prime}} .
\end{gathered}
$$

We numerically calculate $\alpha_{n}$ and $\beta_{n}$ using the excited states $\psi_{z, n}$ obtained in Sec. II A. For a circular dot we obtain $\alpha_{n}=\beta_{n}$. For an elliptical dot with realistic parameters, these coefficients remain close to each other since the confinement along $\hat{z}$ in quantum dots is always stronger than the in-plane confinements. Table I shows an example for the values of $\alpha_{n}$ and $\beta_{n}$. With the set of parameters used in Table I, we find $x_{0}^{\prime} y_{0}^{\prime} \eta=-8.64 \times 10^{-4} \mathrm{~T}^{-2}$. Therefore the correction including $B_{x} B_{y} x_{0}^{\prime} y_{0}^{\prime} \eta$ in Eq. (27) remains a subleading term so long

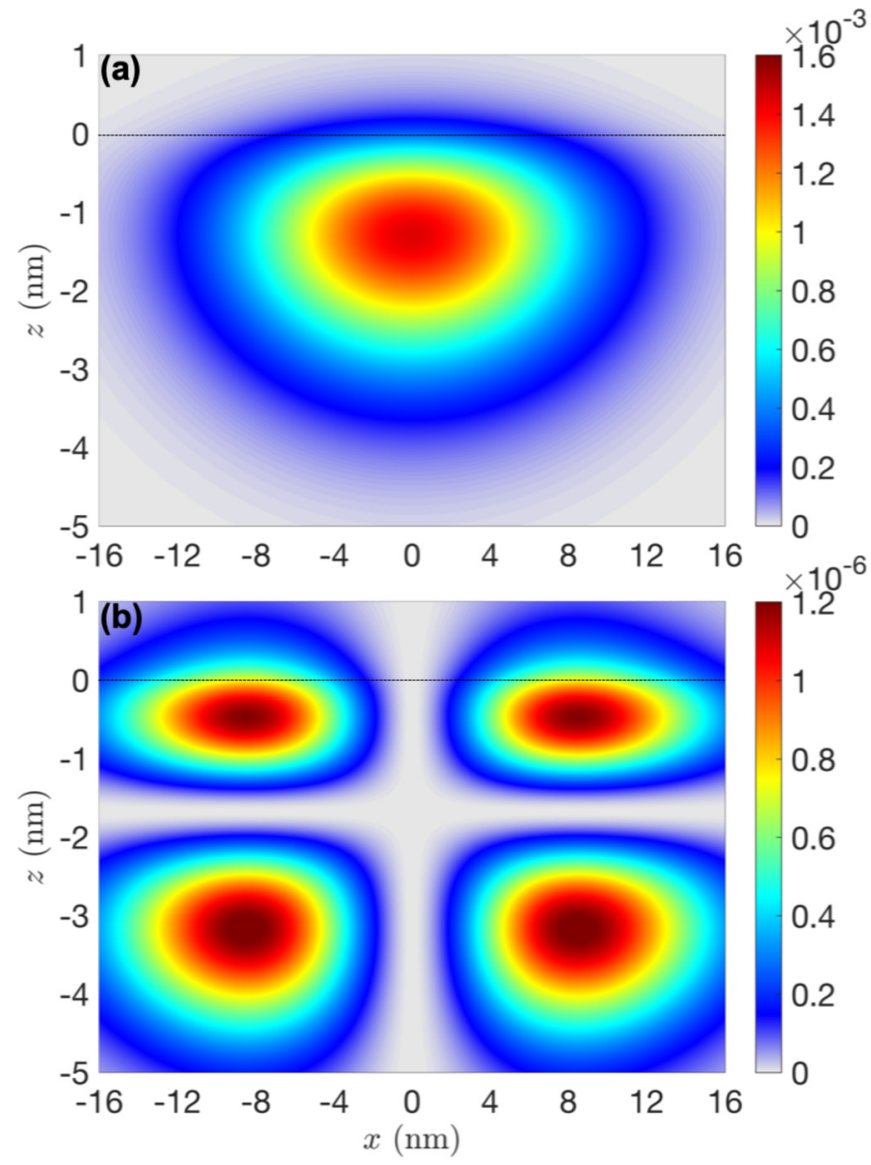

FIG. 4. (a) The electron probability density of a quantum dot with an ideal interface in the $x-z$ plane in leading order, $\left|\psi_{x y z, 0}^{(\mathbf{0})}(\mathbf{B})(x, y=0, z)\right|^{2}\left(1 \mathrm{~nm}^{-3}\right)$; see Eq. (26). (b) The correction to the probability amplitude in the $x-z$ plane, $\mid \psi_{x y z}^{\|}(\mathbf{B})(x, y=$ $0, z)\left.\right|^{2}\left(1 / \mathrm{nm}^{3}\right)$ due to an in-plane magnetic field; see Eq. (27). The parameters used are the same as given in the caption of Table I. The dashed line in both panels marks the ideally flat $\mathrm{Si} / \mathrm{SiGe}$ interface.

as $|\mathbf{B}| \lesssim 10 \mathrm{~T}$. In Fig. 4, we show the probability density in the $x-z$ plane in leading order, $\left|\psi_{x y z, 0}^{(\mathbf{0})}(\mathbf{B})\right|^{2}$, as well as the firstorder correction, $\left|\psi_{x y z, 0}^{(\mathbf{1})}(\mathbf{B})\right|^{2}$. In Appendix B, we complement this section by studying the correction to the ground-state energy due to the presence of an in-plane magnetic field.

Note that for $\mathrm{Si} / \mathrm{SiO}_{2}$ heterostructures, the correction to the envelope function would have the same form as given by Eq. (27). In Appendix D, we show an example for the values of $\alpha_{n}$ and $\beta_{n}$ for a $\mathrm{Si} / \mathrm{SiO}_{2}$ heterostructure.

\section{Envelope function with disordered $\mathrm{Si} / \mathrm{SiGe}$ interface}

So far, we have studied structures where the interface between $\mathrm{Si}$ and $\mathrm{SiGe}$ is perfectly flat and is located at $z=-d_{t}$ and $z=0$. However, during the experimental fabrication of $\mathrm{Si}$ qubit nanostructures, the formation of miscuts and steps at the interfaces is highly probable. Such uncontrolled disorder can modify the valley splitting and its phase, and is considered to be the main reason that makes the valley splitting a devicedependent quantity. In Ref. [22], several configurations for the steps at the interface are considered, and in each case, 


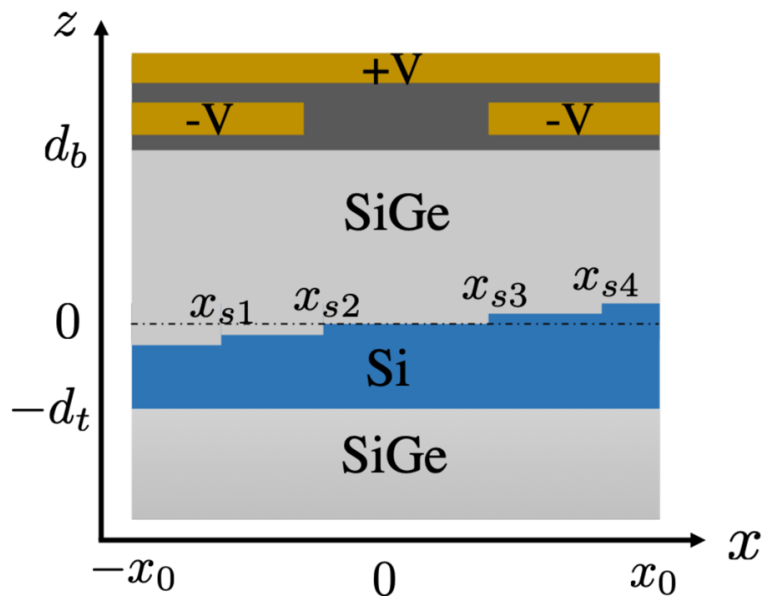

FIG. 5. Schematic layered structure of a quantum dot with stairlike disordered $\mathrm{Si} / \mathrm{SiGe}$ interface.

the envelope function is formed from a variational ansatz that uses a smooth interpolation between the envelope functions far from the step position (i.e., envelope functions for perfect interface).

In this section, we extend our model to include stairlike interface steps. For simplicity, throughout this work, we consider a particular case where the steps are parallel to the $\hat{y}$-axis and are located at the upper $\mathrm{Si} / \mathrm{SiGe}$ interface as depicted in Fig. 5. Our main objective here is to study how these miscuts influence the quantum dot envelope wave function. Note that disorder could also be present at the lower $\mathrm{SiGe} / \mathrm{Si}$ interface. However, since the amplitude of the envelope function is small at the lower interface, the effects of possible disorder is negligible.

In silicon, the thickness of each atomic layer is $a_{0} / 4$ where $a_{0}=0.543 \mathrm{~nm}$ denotes the lattice constant. This indicates that the change in the interface position due to a few miscuts is much smaller than the total thickness of the envelope function along $\hat{z}$ and enables us to use perturbation theory in order to obtain the electron envelope function. We take the $z$ position of the interface layer that contains the quantum dot center as the reference for the position of the barrier interface (e.g., the layer within $\left[x_{2}, x_{3}\right]$ in Fig. 6), and take any change to the interface position due to the miscuts as a perturbation. We describe the disordered $\mathrm{SiGe} / \mathrm{Si} / \mathrm{SiGe}$ interface with the step potential

$$
U_{\text {dis }}(x, z)=U(z)+U_{\text {steps }}(x, z),
$$

where $U(z)$ is given by Eq. (2) and

$$
\begin{aligned}
U_{\text {steps }}(x, z)=U_{0}[ & \theta(-z) \theta\left(z+\frac{a_{0}}{4}\right) \theta\left(x-x_{1}\right) \theta\left(x_{2}-x\right) \\
& +\theta(-z) \theta\left(z+\frac{a_{0}}{2}\right) \theta\left(x_{1}-x\right) \\
& -\theta(z) \theta\left(z-\frac{a_{0}}{4}\right) \theta\left(x-x_{3}\right) \theta\left(x_{4}-x\right) \\
& \left.-\theta(z) \theta\left(z-\frac{a_{0}}{2}\right) \theta\left(x-x_{4}\right)\right]
\end{aligned}
$$

TABLE II. The coefficients $\gamma_{m, n}$. Here we assumed $x_{s 1}=-7$ $\mathrm{nm}, x_{s 2}=-2 \mathrm{~nm}, x_{s 3}=3 \mathrm{~nm}$, and $x_{s 4}=7 \mathrm{~nm}$. The other parameters are the same as given in the caption of Table I. We also find $\gamma_{1,0}^{\prime}=0.3784$ and $\gamma_{2,0}^{\prime}=0.0663$; see Eq. (41).

\begin{tabular}{lrrrr}
\hline \hline$m$ & \multicolumn{1}{c}{$\gamma_{m, 0}$} & \multicolumn{1}{c}{$\gamma_{m, 1}$} & \multicolumn{1}{c}{$\gamma_{m, 2}$} & \multicolumn{1}{c}{$\gamma_{m, 3}$} \\
\hline 0 & $\mathrm{~N} / \mathrm{A}$ & 0.0170 & -0.0082 & 0.0047 \\
1 & 0.4204 & -0.0564 & 0.0319 & -0.0215 \\
2 & -0.0353 & 0.0073 & -0.0036 & 0.0019 \\
3 & -0.0214 & 0.0069 & -0.0043 & 0.0031 \\
4 & 0.0088 & -0.0029 & 0.0015 & -0.0008 \\
5 & -0.0001 & -0.0001 & 0.0001 & -0.0001 \\
6 & -0.0025 & 0.0010 & -0.0006 & 0.0003 \\
7 & 0.0025 & -0.0012 & 0.0008 & -0.0006 \\
8 & 0.0004 & -0.0002 & 0.0001 & 0.0000 \\
\hline \hline
\end{tabular}

The Hamiltonian describing the envelope function with disordered interface at finite in-plane magnetic field can again be written in the form of Eq. (16) where, in this case, Eq. (32) is added to the perturbative part of the Hamiltonian, Eq. (18). The ground-state envelope function then reads up to the second-order perturbation with respect to the interface disorders,

$$
\Psi_{x y z, 0}^{\mathrm{dis}}(\mathbf{B})=N_{0}\left[\psi_{x y z, 0}^{(\mathbf{0})}(\mathbf{B})+\psi_{x y z}^{\|}(\mathbf{B})+\psi_{x y z}^{\mathrm{steps}}(\mathbf{B})\right]
$$

where

$$
\psi_{x y z}^{\text {steps }}=\mathcal{D}_{x y z}^{(\mathbf{1})}(\mathbf{B})+\mathcal{D}_{x y z}^{(\mathbf{2})}(\mathbf{B}) .
$$

Here, $N_{0}$ is a normalization constant and $\mathcal{D}_{x y z}^{(\mathbf{1})}$ is the first-order correction due to the interface disorder that amounts to

$$
\mathcal{D}_{x y z}^{(\mathbf{1})}(\mathbf{B})=\psi_{y, 0}\left(B_{x}\right) \sum_{\{m, n\} \neq\{0,0\}} \gamma_{m, n} \psi_{x, m}\left(B_{y}\right) \psi_{z, n},
$$

for which the coefficients

$$
\gamma_{m, n}=\frac{\left\langle\psi_{x, m}\left(B_{y}\right) \psi_{z, n}\left|U_{\text {steps }}\right| \psi_{x, 0}\left(B_{y}\right) \psi_{z, 0}\right\rangle}{E_{0, z}-E_{n, z}-m \hbar \omega_{x}^{\prime}},
$$

shall be calculated numerically. Table II shows examples for the values of $\gamma_{m, n}$. Since the out-of-plane confinement is much stronger than the in-plane confinement, the largest contribution comes from $m=1$ and $n=0$. Moreover, we observe that by taking up to 4 excited states $\psi_{x, m}$, the values of $\gamma_{m, n}$ substantially decay. As such, we can set $m_{\max }=4$ as a cutoff in the summation in Eq. (35).

For the second-order correction due to the interface disorder, we only keep the leading-order terms to arrive at (see Appendix C for more detail),

$$
\begin{aligned}
\mathcal{D}_{x y z}^{(2)} \simeq & c_{1} \psi_{x, 1}\left(B_{y}\right) \psi_{y, 0}\left(B_{x}\right) \psi_{z, 0} \\
& +c_{2} \psi_{x, 2}\left(B_{y}\right) \psi_{y, 0}\left(B_{x}\right) \psi_{z, 0},
\end{aligned}
$$

where the perturbative coefficients $c_{1}$ and $c_{2}$ are given by

$$
\begin{aligned}
c_{1}=\gamma_{1,0}\left[\frac{\left\langle\psi_{x, 0}\left(B_{y}\right) \psi_{z, 0}\left|U_{\text {steps }}\right| \psi_{x, 0}\left(B_{y}\right) \psi_{z, 0}\right\rangle}{\hbar \omega_{x}^{\prime}}\right. \\
\left.-\frac{\left\langle\psi_{x, 1}\left(B_{y}\right) \psi_{z, 0}\left|U_{\text {steps }}\right| \psi_{x, 1}\left(B_{y}\right) \psi_{z, 0}\right\rangle}{\hbar \omega_{x}^{\prime}}\right],
\end{aligned}
$$




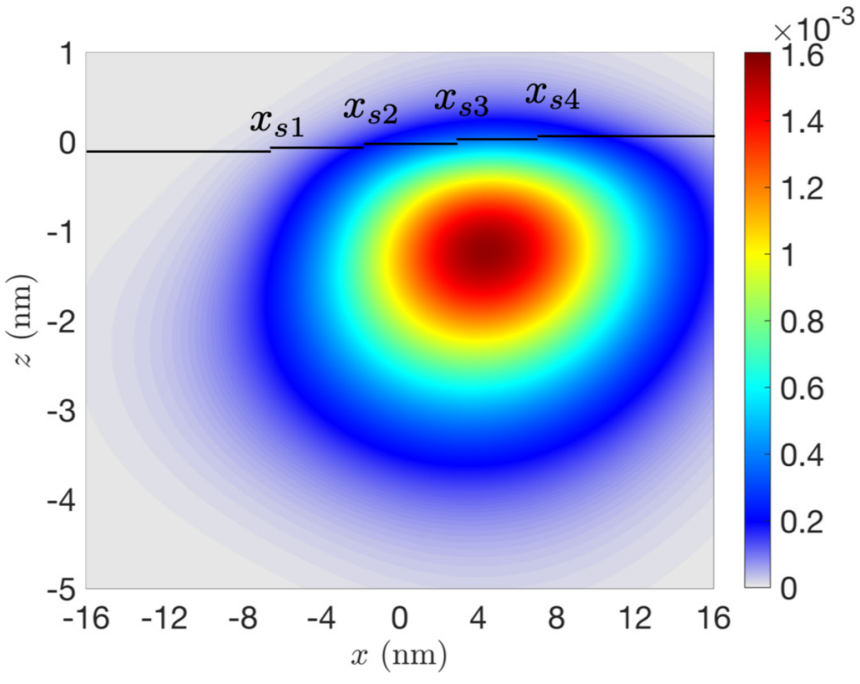

FIG. 6. The electron probability density in the $x-z$ plane, $\left|\Psi_{x y z, 0}^{\mathrm{dis}}(\mathbf{B})(x, y=0, z)\right|^{2}\left(1 / \mathrm{nm}^{3}\right)$, for a quantum dot with disordered interface. The locations of the interface steps are given in the caption of Table II and the other parameters are the same as given in the caption of Table I. The solid lines mark the disordered $\mathrm{Si} / \mathrm{SiGe}$ interface.

$$
c_{2}=-\gamma_{1,0} \frac{\left\langle\psi_{x, 2}\left(B_{y}\right) \psi_{z, 0}\left|U_{\text {steps }}\right| \psi_{x, 1}\left(B_{y}\right) \psi_{z, 0}\right\rangle}{\hbar \omega_{x}^{\prime}} .
$$

Therefore we find

$$
\psi_{x y z}^{\text {steps }}(\mathbf{B})=\psi_{y, 0}\left(B_{x}\right) \sum_{\{m, n\} \neq\{0,0\}} \gamma_{m, n}^{\prime} \psi_{x, m}\left(B_{y}\right) \psi_{z, n},
$$

in which the perturbative coefficients become

$$
\gamma_{m, n}^{\prime}= \begin{cases}\gamma_{m, n}+c_{1}, & \{m, n\}=\{1,0\} \\ \gamma_{m, n}+c_{2}, & \{m, n\}=\{2,0\} \\ \gamma_{m, n}, & \text { otherwise }\end{cases}
$$

In Fig. 6, we show the electron probability density in the $x-z$ plane in the presence of interface steps. The asymmetry around $x=0$ in this case is due to the change of the quantum-dot thickness due to the interface disorder. Since in our model the Si quantum well is thicker for $x>0$, the peak of the probability density is also shifted towards $x>0$. In Appendix B, we study the correction to the ground-state energy due to the presence of the interface steps.

We point out here that other disorder configurations can also be analyzed using the similar perturbative approach that we presented in this section. In particular, if the interface steps are not parallel to the $\hat{y}$ axis, it is easy to see that Eqs. (35) and (37) have to be generalized to include the in-plane excited states along $\hat{y}, \psi_{y, m^{\prime}}$. In the next section, we use the envelope functions we found in this section to study and discuss how the valley splitting of a quantum dot depends on the electric and magnetic fields for an ideally flat as well as disordered $\mathrm{Si} / \mathrm{SiGe}$ interfaces.

\section{DISCUSSION}

Within the effective mass theory, the two low-lying valley components of the quantum dot can be written as

$$
\begin{aligned}
& |+z\rangle=\Psi_{x y z, 0}(\mathbf{B}) e^{i k_{0} z} u_{+z}(\mathbf{r}), \\
& |-z\rangle=\Psi_{x y z, 0}(\mathbf{B}) e^{-i k_{0} z} u_{-z}(\mathbf{r}) .
\end{aligned}
$$

Here, $k_{0} \simeq 0.85\left(2 \pi / a_{0}\right)$ describes the Bloch wave vector of the conduction band minima and $u_{ \pm z}(r)$ are the periodic parts of the Bloch functions for the $\pm z$ valleys in silicon. We express these functions by a plane wave expansion,

$$
u_{ \pm z}(r)=\sum_{\mathbf{G}} C_{ \pm}(\mathbf{G}) e^{i \mathbf{G} . \mathbf{r}}
$$

for which $\mathbf{G}=\left(G_{x}, G_{y}, G_{z}\right)$ is the reciprocal lattice vector. The coefficients in this expansion for the two valleys are related via the time-reversal symmetry relation $C_{-}(\mathbf{G})=$ $C_{+}^{*}(-\mathbf{G})$. The wave vectors and their corresponding coefficients $C_{+}(\mathbf{G})$ for $\mathrm{Si}$ are studied in Ref. [20].

The valley-orbit coupling is given by

$$
\Delta_{v o}=\left\langle+z\left|-e F_{z} z+U(z)\right|-z\right\rangle .
$$

Note that $e F_{z} z=\epsilon_{0} \tilde{z}$, and given $\epsilon_{o} \ll U_{0}$ for all practical values of $F_{z}$ [see Eq. (53)], the valley-orbit coupling is strongly dominated by the matrix element of the interface potential $U(z)$. Indeed, the role of the electric field is to control and shape $\psi_{z}$, and the contributions from the matrix element of $-e F_{z} z$ in the valley-orbit coupling can be neglected [20,30]. The valley splitting is found from the above equation by $E_{v s}=2\left|\Delta_{v o}\right|$ and the valley phase can be found by

$$
\phi_{v}=\tan ^{-1}\left[\operatorname{Im}\left(\Delta_{v o}\right) / \operatorname{Re}\left(\Delta_{v o}\right)\right] .
$$

\section{A. Electrical dependence of the valley splitting for an ideal quantum dot}

In this section, we consider a quantum dot with an ideal interface in the absence of a magnetic field and use the results of Sec. II A to find the electrical and interface-potential dependence of the valley splitting and the valley phase. As explained in Sec. II, since the electric field pushes the envelope function towards the upper $\mathrm{SiGe}$ barrier, the probability density of the ground state at the lower $\mathrm{SiGe} / \mathrm{Si}$ interface is negligible (assuming $F_{z} \geqslant 2 \mathrm{MV} / \mathrm{m}$ for $d_{t}=10 \mathrm{~nm}$.) Therefore we can drop the lower interface potential and only take $U(z)=U_{0} \theta(z)$. The valley-orbit coupling at zero magnetic field for an ideal quantum dot then becomes

$$
\begin{aligned}
\Delta_{v o}^{0}=U_{0} \sum_{\mathbf{G}_{1}, \mathbf{G}_{2}} & {\left[C_{+}^{*}\left(\mathbf{G}_{1}\right) C_{-}\left(\mathbf{G}_{2}\right)\right.} \\
& \left.\times \int_{-\infty}^{+\infty} e^{-i\left(\mathbf{G}_{1}-\mathbf{G}_{2}+2 k_{0}\right) z} \theta(z) \psi_{z, 0}^{2} d z\right] .
\end{aligned}
$$

To carry on, we note that the terms with $\mathbf{G}_{1} \neq \mathbf{G}_{2}$ would lead to fast oscillations in the integrand that average to zero. We 
therefore only consider terms with $\mathbf{G}_{1}=\mathbf{G}_{2}$ and define,

$$
\mathcal{C}_{0}=\sum_{\mathbf{G}} C_{+}^{*}(\mathbf{G}) C_{-}(\mathbf{G})
$$

which we find to be $\mathcal{C}_{0}=-0.2607$ using Ref. [20].

We now take the integration by parts and find for an ideal quantum dot,

$$
\Delta_{v o}^{0}=\Delta_{\text {int }}+\Delta_{t}
$$

Here, $\Delta_{\text {int }}$ is the contribution that comes from the amplitude of $\psi_{z, 0}$ at the $\mathrm{Si} / \mathrm{SiGe}$ interface:

$$
\Delta_{\text {int }}=-i \frac{U_{0} \mathcal{C}_{0}}{2 k_{0} z_{0}} \int_{-\infty}^{+\infty} e^{-2 i k_{0} z} \delta(z) \psi_{z, 0}^{2} d z,
$$

and $\Delta_{t}$ is the contribution that originates from the tail of $\psi_{z, 0}$ inside the barrier,

$$
\Delta_{t}=-i \frac{U_{0} \mathcal{C}_{0}}{2 k_{0} z_{0}} \int_{-\infty}^{+\infty} e^{-2 i k_{0} z} \theta(z) 2 \psi_{z, 0} \psi_{z, 0}^{\prime} d z .
$$

In order to find analytical expressions for these contributions, we use Eqs. (14) and (15) and also use the expansions given by Eqs. (A9) (note that by using these equations, we assume $U_{0} \gg \epsilon_{0}$ which is valid for all relevant valued for the electric field $F_{z}$, see Eq. (53)]. We finally arrive at the result

$$
\Delta_{\text {int }}=-i \mathcal{C}_{0} \frac{e F_{z}}{2 k_{0}}
$$

and

$$
\Delta_{t}=-\Delta_{\text {int }}\left[1-\frac{1}{2 \tilde{U}_{0}}+i \frac{k_{0} z_{0}}{\sqrt{\tilde{U}_{0}}}\right]^{-1} .
$$

The last term in the square bracket is a number larger than 1 (having $F_{z}=2$ to $20 \mathrm{MV} / \mathrm{m}$ for a SiGe barrier, we find $k_{0} z_{0} / \sqrt{\tilde{U}_{0}} \gtrsim 5$ ). This indicates that $\left|\Delta_{\text {int }}\right|$ is larger than $\left|\Delta_{t}\right|$ (by nearly a factor of 6.) In Appendix D, we study how Eq. (52) is modified in a $\mathrm{Si} / \mathrm{SiO}_{2}$ heterostructure, see Eq. (D1).

We conclude that the valley-orbit coupling (and hence also the valley splitting) within the leading order scales linearly with the electric field while it is independent of the interface potential (as long as $U_{0} \gg \epsilon_{0}$ ). This linear dependence is experimentally observed in Ref. [3] for a $\mathrm{SiO}_{2}$ barrier (that has a much stronger interface potential $U_{0}=3 \mathrm{eV}$ compared with $\mathrm{SiGe}$ ) and it is also predicted from a theory analysis assuming that the envelope function has zero amplitude inside the barrier [23].

In addition to the linear-in-electric-field term, here we find the valley splitting also has a small nonlinear dependence on the electric field (note that $1 / \tilde{U}_{0} \propto F_{z}^{2 / 3}$ ). This nonlinear contribution originates from the penetration of the envelope function into the barrier, and can be neglected so long as $\tilde{U}_{0} \gg 1$; this holds provided

$$
F_{z} \ll U_{0} \frac{\sqrt{2 m_{l} U_{0}}}{e \hbar} .
$$

For a SiGe barrier with $U_{0}=150 \mathrm{meV}$, the right side of the above inequality becomes $\sim 280 \mathrm{MV} / \mathrm{m}$. This essentially means for all practically relevant values for $F_{z}$, the valley splitting remains a linear function of the electric field (note

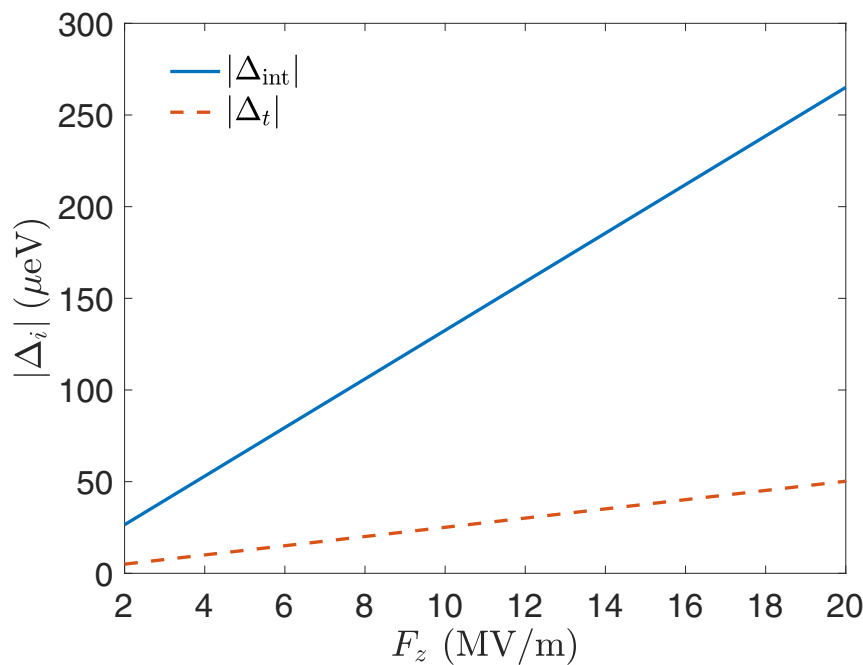

FIG. 7. $\left|\Delta_{\text {int }}\right|$ and $\left|\Delta_{t}\right|$ contributions to the valley-orbit coupling for a SiGe barrier. As explained in the main text, the dominant contribution to the valley-orbit coupling comes from the amplitude of the envelope function at the barrier interface described by $\left|\Delta_{\text {int }}\right|$.

that the electrical breakdown field is reported to be $30 \mathrm{MV} / \mathrm{m}$ for $\mathrm{Si}$ and $25 \mathrm{MV} / \mathrm{m}$ for $\mathrm{Si}_{0.75} \mathrm{Ge}_{0.25}$ [32].)

In Fig. 7, we used Eqs. (51) and (52) and show $\left|\Delta_{\text {int }}\right|$ and $\left|\Delta_{t}\right|$ as a function of the electric field $F_{z}$. The values that we show in the figure are in agreement with Refs. [21] and [30] where $\left|\Delta_{v o}\right| \sim 200 \mu \mathrm{eV}$ is reported for $F_{z}=15 \mathrm{MV} / \mathrm{m}$.

Finally, from Eq. (51) and (52), we find that the valley phase of a quantum dot with perfect interface up to the leading order only depends on the interface potential,

$$
\phi_{v} \simeq \tan ^{-1}\left[\frac{-\hbar k_{0}}{\sqrt{2 m_{l} U_{0}}}\right],
$$

that becomes $\phi_{v} \simeq-0.44 \pi$ for a SiGe barrier.

We close this section by noting that Eqs. (51) and (52) indicate that the valley-orbit coupling for an ideal quantum dot does not depend on the silicon layer thickness, $d_{t}$. This is a consequence of our initial assumption that the envelope function has a negligible amplitude at the lower $\mathrm{SiGe} / \mathrm{Si}$ interface due to the electric field. At the limit where $F_{z} \rightarrow 0$, this assumption clearly breaks down, and both lower and higher interfaces become equally important. In this case, it has been shown, using both the effective mass approximation as well as the tight-binding method, that the valley splitting oscillates as a function of the silicon layer thickness [19,33]. We also note that the electric field $F_{z}$ is, in turn, influenced by the electron density. References [34] and [35] include a direct measurement of the valley splitting as a function of the electron density.

\section{B. Magnetic dependence of the valley split ting for an ideal quantum dot}

We now extend the results of the last section by including an in-plane magnetic field. As we have shown in Sec. II B, the electron envelope function $\Psi_{x y z, 0}$ in the presence of an inplane magnetic field includes excited states of the out-of-plane motion $\psi_{z, n}$, see Eq. (25) and (27). Compared to the ground 


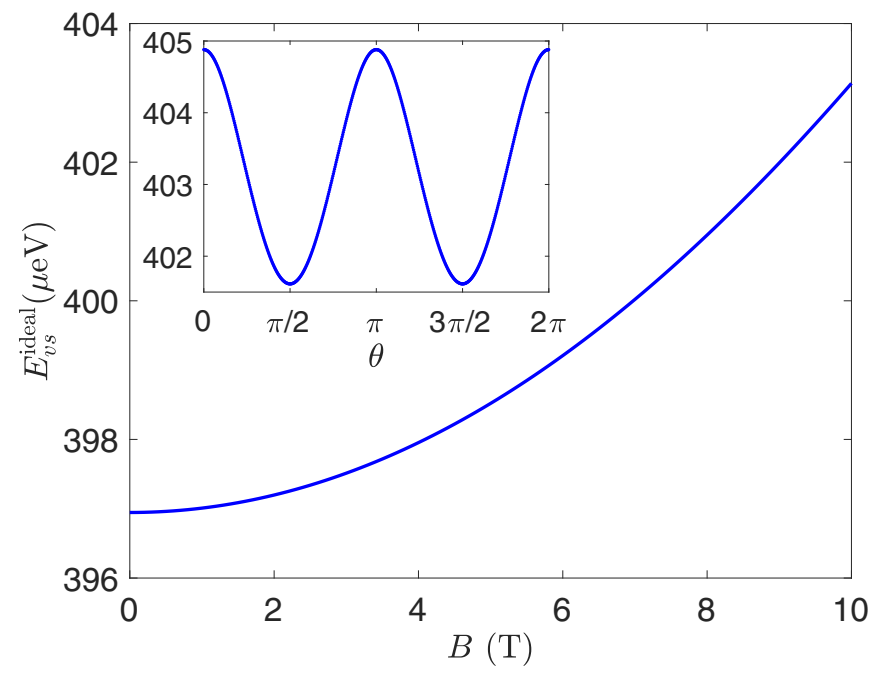

FIG. 8. The valley splitting for a quantum dot with ideal interface at $F_{z}=15 \mathrm{MV} / \mathrm{m}$ as a function of magnetic field. Here we have taken $\mathbf{B}=B(\cos (\theta), \sin (\theta), 0)$ and set $\theta=\pi / 4$. The size of the quantum dot is the same as indicated in the caption of Table I: $x_{0}=12 \mathrm{~nm}$ and $y_{0}=15 \mathrm{~nm}$. (Inset) The valley splitting at $B=10 \mathrm{~T}$ as a function of direction of the magnetic field.

state $\psi_{z, 0}$, the excited states can have a larger amplitude at the $\mathrm{Si} / \mathrm{SiGe}$ interface, and penetrate further to the barrier. As such, we generally expect that the valley splitting should increase in an in-plane magnetic field. In addition, as one can see from Fig. 3, depending on the electric field, the excited states can have a sizable amplitude at the lower $\mathrm{SiGe} / \mathrm{Si}$ interface $z=-d_{t}$. Therefore we take into account both upper and lower interfaces and consider a barrier potential of the form $U(z)=U_{0} \theta(z)+U_{0} \theta\left(-z-d_{t}\right)$. We use Eqs. (25) and (27) and find for the valley-orbit coupling:

$$
\begin{aligned}
& \Delta_{v o}^{\text {ideal }}\left(\mathbf{B}, F_{z}\right)=\Delta_{v o}^{0}\left[1+B_{x}^{2} B_{y}^{2} x_{0}^{\prime 2} y_{0}^{\prime 2} \eta^{2}\right] \\
& +\mathcal{C}_{0} B_{x}^{2}\left(\frac{y_{0}^{\prime}}{z_{0}}\right)^{2} \sum_{n, n^{\prime}} \alpha_{n} \alpha_{n^{\prime}} \int_{-\infty}^{\infty} e^{-2 i k_{0} z} \psi_{z, n} U(z) \psi_{z, n^{\prime}} d z \\
& +\mathcal{C}_{0} B_{y}^{2}\left(\frac{x_{0}^{\prime}}{z_{0}}\right)^{2} \sum_{n, n^{\prime}} \beta_{n} \beta_{n^{\prime}} \int_{-\infty}^{\infty} e^{-2 i k_{0} z} \psi_{z, n} U(z) \psi_{z, n^{\prime}} d z
\end{aligned}
$$

Using the excited states $\psi_{z, n}$ from Sec. II A, we numerically calculate the above integrals. The coefficients $\alpha_{n}, \beta_{n}$ and $\eta$ are introduced in Sec. II B and as we explained there, up to a few Tesla, the terms containing $\alpha$ and $\beta$ are strongly dominant over the correction containing $\eta$. Therefore, to the leading order, the magnetic contribution to the valley splitting scales quadratically with the magnetic field. Moreover, at finite magnetic fields, the valley splitting becomes dependent on ratio of the lateral confinement to the electrical confinement, $x_{0}^{\prime} / z_{0}$ and $y_{0}^{\prime} / z_{0}$, see Eqs. (23) and (24).

Equation (55) indicates that for an elliptical quantum dot at a fixed magnetic field, the valley splitting reaches its maximal value when the direction of the magnetic field is perpendicular to the axis with the larger radius. In the main plot of Fig. 8, we show the valley splitting as a function of magnetic field at a fixed direction. In the inset plot of the figure, we show the valley splitting as a function of the direction of the field. We observe that the valley splitting for a quantum dot with an ideal interface only slightly increases with the magnetic field; even by having a magnetic field as large as $|\mathbf{B}|=10 \mathrm{~T}$, the increase in valley splitting is only nearly $2 \%$ whereas the deviation in the valley splitting due to changing the direction of the field (at the used set of parameters) is only around 1\% (note that for a circular quantum dot, $x_{0}=y_{0}$, changing the direction of the magnetic field does not change the valley splitting due to the symmetry of the system). This also indicates that the dominant contribution to the valley splitting remains a linear function of the electric field $F_{z}$ at the finite magnetic fields.

\section{Valley splitting of a quantum dot with disordered interface}

We now consider a realistic quantum dot with miscuts and steps at the $\mathrm{Si} / \mathrm{SiGe}$ interface and aim to study the valley splitting and its electromagnetic dependence. We take the interface potential given by Eq. (31) and use the resulting envelope function Eq. (33). We then find for the valley-orbit coupling,

$$
\Delta_{v o}^{\text {dis }}=N_{0}^{2}\left[\Delta_{v o}^{\text {ideal }}+\Delta_{s}+\Delta_{(\mathbf{1})}+\Delta_{(\mathbf{2})}\right],
$$

where $\Delta_{v o}^{\text {ideal }}$ is the valley-orbit coupling for an ideal interface given by Eq. (55), $\Delta_{s}$ is the largest contribution originating from the interface disorders and reads

$$
\Delta_{s}=\mathcal{C}_{0} \int_{-\infty}^{\infty} e^{-2 i k_{0} z} \psi_{x, 0}^{2}\left(B_{y}\right) \psi_{z, 0}^{2} U_{\text {steps }} d x d z,
$$

and $\Delta_{(\mathbf{1})}$ is a contribution that is first order with respect to $\mathcal{D}_{x y z, 0}^{\prime}$ and reads

$$
\Delta_{(\mathbf{1})}=\sum_{m, n} \Delta_{(\mathbf{1}),\{m, n\}}=2 \mathcal{C}_{0} \sum_{m, n} \gamma_{m, n}^{\prime} f_{m, n},
$$

where

$$
\begin{aligned}
f_{m, n}=\int_{-\infty}^{\infty}[ & e^{-2 i k_{0} z} \psi_{x, m}\left(B_{y}\right) \psi_{z, n} \\
& \left.\times U_{\text {steps }} \psi_{x, 0}\left(B_{y}\right) \psi_{z, 0}\right] d x d z .
\end{aligned}
$$

The last term, $\Delta_{(\mathbf{2})}$, is a small and sub-leading contribution that is second-order with respect to $\mathcal{D}_{x y z, 0}^{\prime}$ and $\psi_{x y z, 0}^{(\mathbf{1})}$. More details on this term can be found in the Appendix E.

Note that, in general, we have $\left|\Delta_{(\mathbf{2})}\right| \ll\left|\Delta_{(\mathbf{1})}\right|$ and $\left|\Delta_{(\mathbf{1})}\right|<$ $\left|\Delta_{v o}\right|,\left|\Delta_{s}\right|$. However, as we discuss below, depending on the number and location of interface steps, $\left|\Delta_{v o}+\Delta_{s}\right|$ can become a small number. In this case, the contribution from $\Delta_{(\mathbf{1})}$ becomes (more) important in determining the valley splitting and its phase.

\section{A single step at the interface}

Let us now study the structure and effects of $\Delta_{s}$ and $\Delta_{(\mathbf{1})}$. We begin by considering the simplest case; that is, when there is only a single step at the interface. We take the width of the step to be $+a / 4$; the step potential is then obtained from Eq. (32) by taking $x_{s 4} \rightarrow+\infty$ and $x_{s 1}, x_{s 2} \rightarrow-\infty$. We then take $x_{s 3}=x_{s} \geqslant 0$ to be the position of the only interface step. From Eq. (57) for a quantum dot with a single interface step, 

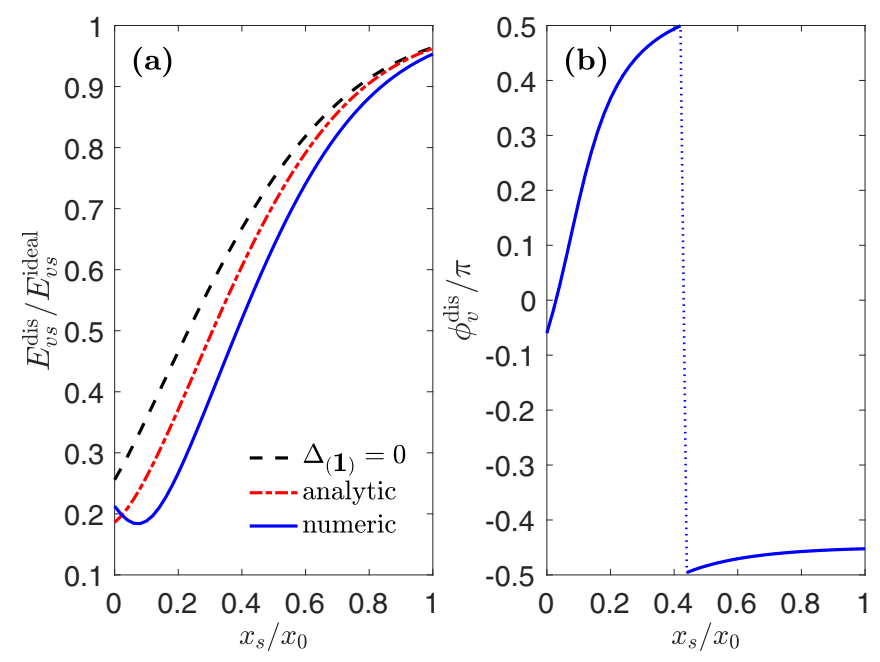

FIG. 9. (a) The normalized valley splitting for a quantum dot with single interface step as a function of the step location. The dash-dotted line is found by using analytic relations Eqs. (48), (60), and (61) and taking $N_{0}=1$. The solid and dashed lines are deduced from numerical calculation. (b) The valley phase of a quantum dot with a single interface step as a function of the step location. Both panels are obtained at at $B=0$ and $F_{z}=15 \mathrm{MV} / \mathrm{m}$.

we obtain

$$
\begin{aligned}
\Delta_{s}^{1 s}\left(x_{s}\right) \simeq & -\frac{1}{2} \Delta_{v o}^{0} \operatorname{Erfc}\left(\sqrt{2} x_{s} / x_{0}^{\prime}\left(B_{y}\right)\right) \\
& \times\left[1-e^{-\left(a_{0} / 2 z_{0}\right)\left(\sqrt{\tilde{U}_{0}}+i k_{0} z_{0}\right)}\right] .
\end{aligned}
$$

In order to analyze $\Delta_{(\mathbf{1})}^{1 s}$, we note that a complete assessment of this term requires numerical calculations. However, we can obtain a rough estimation by only considering the largest contribution to Eq. (58), i.e., the term corresponding to $m=1$ and $n=0$. For further simplicity, we also drop the second-order correction to the envelope function, $\mathcal{D}_{x y z, 0}^{(2)}$, so that we take $\gamma_{1,0}^{\prime}=\gamma_{1,0}$ and $N_{0}=1$. We then arrive at the largest contribution to $\Delta_{(\mathbf{1})}$ due to $m=1$ and $n=0$,

$$
\begin{aligned}
\Delta_{(\mathbf{1}),\{1,0\}}^{1 s}\left(x_{s}\right) \simeq- & \gamma_{1,0} \Delta_{v o}^{0} \sqrt{\frac{2}{\pi}} e^{-2\left(x_{s} / x_{0}^{\prime}\left(B_{y}\right)\right)^{2}} \\
\times & {\left[1-e^{-\left(a_{0} / 2 z_{0}\right)\left(\sqrt{\tilde{U}_{0}}+i k_{0} z_{0}\right)}\right], }
\end{aligned}
$$

in which

$$
\begin{aligned}
\gamma_{1,0}= & \frac{\epsilon_{0}}{\hbar \omega_{x}^{\prime}\left(B_{y}\right)} \frac{1}{2 \sqrt{2 \pi}} e^{-2\left(x_{s} / x_{0}^{\prime}\left(B_{y}\right)\right)^{2}} \\
& \times \frac{1}{\sqrt{\tilde{U}_{0}}}\left[1-e^{-\left(a / 2 z_{0}\right) \sqrt{\tilde{U}_{0}}}\right] .
\end{aligned}
$$

$\Delta_{s}^{1 s}\left(x_{s}\right)$ and $\Delta_{(\mathbf{1}),\{1,0\}}^{1 s}\left(x_{s}\right)$ are clearly out of phase with $\Delta_{v o}^{\text {ideal }}$. This can significantly modify and suppress the valley splitting. Note that $\Delta_{s}^{1 s}\left(x_{s}\right)$ and $\Delta_{(\mathbf{1}),\{1,0\}}^{1 s}\left(x_{s}\right)$ are monotonically decreasing if the interface step is located further away from the quantum-dot center.

In Fig. 9(a), we present the normalized valley splitting as a function of the step location at $B=0$. By neglecting $\Delta_{(\mathbf{1})}$ in the valley-orbit coupling (shown in the figure by the dashed black line), we observe, as predicted, that the valley splitting is monotonically decreasing as the interface step moves closer to the quantum dot center. At $x=0$, the valley splitting is suppressed by $75 \%$. This amount of suppression is reported in Ref. [22] where a variational ansatz is used to approximate the envelope function in the presence of a single step. By taking into account the $\Delta_{(\mathbf{1}),\{1,0\}}^{1 s}\left(x_{s}\right)$ contribution (shown by the dash-dotted red line), we observe that the valley splitting only becomes further suppressed.

Remarkably, if we numerically calculate $\Delta_{(\mathbf{1})}^{1 s}$ from Eq. (58) and take into account not only the dominant term corresponding to $m=1$ and $n=0, \Delta_{(\mathbf{1}),\{1,0\}}^{1 s}$, but also the other terms due to the out-of-plane excited states, $\Delta_{(\mathbf{1}),\{m, n \geqslant 1\}}^{1 s}$, we observe that the valley splitting has in fact a nonmonotonic behavior as a function of the distance between the single interface step and the quantum dot center. Indeed, the terms $\Delta_{v o}^{\text {ideal }}$, $\Delta_{s}^{1 s}$, and $\Delta_{(\mathbf{1}),\{1,0\}}^{1 s}$ are originating from the ground state of the out-of-plane motion, $\psi_{z, 0}$. As it is already shown, the closer the step to the quantum dot center, the smaller the sum $\left|\Delta_{v o}^{\text {ideal }}+\Delta_{s}^{1 s}+\Delta_{(\mathbf{1}),\{1,0\}}^{1 s}\right|$ becomes. At the same time, placing the step closer to the center would increase $\left|\Delta_{(\mathbf{1}),\{m, n \geqslant 1\}}^{1 s}\right|$ (due to the increase of the coefficients $\gamma_{m, n \geqslant 1}$ ) until at some point the contribution from $\Delta_{(\mathbf{1}),\{m, n \geqslant 1\}}^{1 s}$ becomes the dominant term in the total valley splitting. When the interface step is placed any closer to the quantum dot center, the valley splitting then begins to rise. In Appendix F, we study this effect in detail and show how considering the term $\Delta_{(\mathbf{1}),\{m, n \geqslant 1\}}^{1 s}$ would influence the real and imaginary parts of the total valley-orbit coupling, $\Delta_{v o}^{\text {dis }}$. It turns out that at the step location where the valley splitting reaches to its minimum, $x_{s}=x_{m}^{1 s}$, we have

$$
\operatorname{Re}\left[\Delta_{v o}^{\mathrm{dis}}\left(x_{s}=x_{m}^{1 s}\right)\right]=\operatorname{Im}\left[\Delta_{v o}^{\mathrm{dis}}\left(x_{s}=x_{m}^{1 s}\right)\right] .
$$

At $x_{s}<x_{m}^{1 s}$, the dominant contribution to the $\Delta_{v o}^{\text {dis }}$ becomes a real number while $\operatorname{Re}\left[\Delta_{v o}^{\mathrm{dis}}\right]$ increases by placing the step closer to the center. At $x_{s}>x_{m}^{1 s}$, the dominant contribution to the $\Delta_{v o}^{\text {dis }}$ becomes an imaginary number while $\operatorname{Im}\left[\Delta_{v o}^{\text {dis }}\right]$ increases by placing the step further away from the quantum dot center. The nonmonotonic behavior of the valley splitting as a function of the location of a single interface step is also predicted in Ref. [36] where the authors use a tight-binding method.

In Fig. 9(b), we have shown the valley phase as a function of the step location. Note that the valley phase, given by Eq. (45), is a $\pi$-periodic function defined within $[-\pi / 2, \pi / 2]$. Therefore the sudden jump of the valley phase that occurs at $x_{s} \sim 0.45 x_{0}$ can be removed by subtracting $\pi$ from the values above the jump.

Let us now study how the valley splitting scales with the electric and magnetic fields. As we have shown in Sec. III A, the valley-orbit coupling for an ideal quantum dot $\Delta_{v o}^{0}$ is a linear function of the electric field. As such, the $\Delta_{s}^{1 s}$ term given by Eq. (60) is also linear in electric field. However, $\Delta_{(\mathbf{1})}^{1 s}$ is a nonlinear function with respect to the electric field. Given Eq. (62), the dominant coefficient $\gamma_{1,0}$ scales linearly with the electric field, and this gives rise to a quadratic scaling of $\Delta_{(\mathbf{1}),\{1,0\}}^{1 s}$ with the electric field (more accurately, if we keep $c_{1}$ in Eq. (41), it is easy to show that $\Delta_{(\mathbf{1}),\{1,0\}}^{1 s}$ acquires one more term that is cubic in the electric field.) 

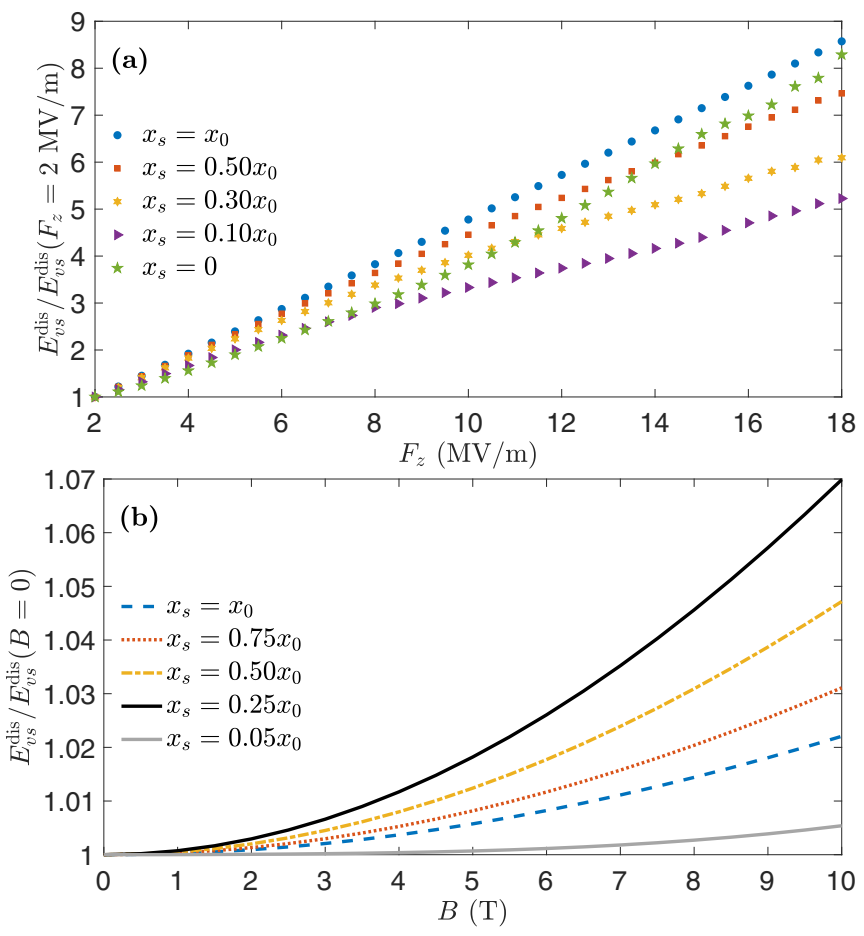

FIG. 10. (a) The normalized valley splitting for a quantum dot with a single interface step for various step location at $B=0$, as a function of the electric field strength. (b) The normalized valley splitting as a function of the in-plane magnetic field. Here we assumed $\mathbf{B}=B(\cos \pi / 4, \sin \pi / 4,0)$ and $F_{z}=15 \mathrm{MV} / \mathrm{m}$.

Therefore, in general, the valley splitting for a quantum dot with a single interface is a nonlinear function of the electric field due to the $\Delta_{(\mathbf{1})}$ term as well as the normalization constant $N_{0} \simeq\left[1+\sum_{m, n} \gamma_{m, n}^{2}\right]^{-1 / 2}$. In Fig. 10(a), we show the valley splitting as a function of the electric field for several locations for the single interface step.

Since $\Delta_{(\mathbf{1})}$ grows with the electric field faster than a linear function, for $x_{s}>x_{m}^{1 s}$, we expect the valley splitting should scale sub-linearly by the electric field. The further the step is located away from the center, the smaller $\Delta_{(\mathbf{1})}$ becomes so that the valley splitting approaches being a linear function of the electric field. For $x_{s}<x_{m}^{1 s}$, the valley splitting is mainly determined from the terms with $n \geqslant 1$ in $\Delta_{(\mathbf{1})}$. Therefore numerical analysis is required to find the dependency of the valley splitting on the electric field.

In order to understand how the valley splitting changes with an in-plane magnetic field, we note that the confinement length $x_{0}^{\prime}\left(B_{y}\right)$ is reduced by the magnetic field. Using Eqs. (23), the effect of the magnetic field to the evolution of $\Delta_{s}^{1 s}\left(x_{s}\right)$ and $\Delta_{(\mathbf{1}),\{1,0\}}^{1 s}\left(x_{s}\right)$ is equivalent as if $x_{0}^{\prime}=x_{0}$ and $x_{s}$ is located from the center at a larger distance,

$$
x_{s} \rightarrow x_{s}\left(1+\frac{1}{4} \frac{m_{t}}{m_{l}} \frac{x_{0}^{4}}{l_{B_{y}}^{4}}\right)^{1 / 4} .
$$

Therefore we expect that the magnetic field should always increase the valley splitting if $x_{s}>x_{m}$. Since the magnetic field effectively increases $x_{s}$, the increase of the valley splitting by the magnetic field is larger at the step location where the slope of the curve given in the panel (a) of Fig. 9 is steeper. For $x_{s}<x_{m}$, the magnetic field decreases $\Delta_{(\mathbf{1}),\{m, n \geqslant 1\}}^{1 s}\left(x_{s}\right)$ that controls the valley splitting. However, we observe that the valley splitting still slightly increases as a function of the magnetic field due to the increase of $\Delta_{v o}^{\text {ideal }}+\Delta_{s}^{1 s}$ with the magnetic field. We display the valley splitting as a function of the in-plane magnetic field in the panel (b) of Fig. 10

\section{Two steps at the interface}

We now consider a quantum dot with two interface steps having widths $-a_{0} / 4$ and $a_{0} / 4$. The step potential is then obtained from Eq. (32) by taking $x_{s 1} \rightarrow-\infty$ and $x_{s 4} \rightarrow+\infty$. For further simplification, we also assume that the two steps are placed symmetrically around the center so that we can write $-x_{s 2}=x_{s 3}=x_{s}$. In this case, finding the contribution from $\Delta_{(\mathbf{1})}$ requires numerical analysis (even only for the term with $m=1$ and $n=1$ in Eq. (58).) However, we can still obtain qualitative understanding of the behavior of the valley splitting by only considering the effect of $\Delta_{s}$. In order to arrive to the extension of Eq. (57) for a quantum dot with two symmetrically located steps, we integrate over $z$ by parts, similar to Sec. III A, and neglect the small integral containing $\psi_{z, 0}^{\prime}$. With this, we arrive at

$$
\begin{aligned}
\Delta_{s}^{2 s s}\left(x_{s}\right) \simeq & -\frac{1}{2} \Delta_{\text {int }} \operatorname{Erfc}\left(\sqrt{2} x_{s} / x_{0}^{\prime}\left(B_{y}\right)\right) \\
\times & {\left[2-e^{-i k_{0} a_{0} / 2} \psi_{z, 0}\left(\frac{a_{0}}{4 z_{0}}\right)^{2} / \psi_{z, 0}(0)^{2}\right.} \\
& \left.\quad-e^{i k_{0} a_{0} / 2} \psi_{z, 0}\left(-\frac{a_{0}}{4 z_{0}}\right)^{2} / \psi_{z, 0}(0)^{2}\right] .
\end{aligned}
$$

If the steps are located at the center, $x_{s}=0$, we find from the above equation (taking $\left.F_{z}=15 \mathrm{MV} / \mathrm{m}\right) \Delta_{s}^{2 s s}\left(x_{s}=0\right) \simeq$ $(1.93-0.18 i) \Delta_{\text {int }}$ that is larger than $\Delta_{v o}^{\text {ideal }}$ in Eq. (56) [note that $\Delta_{v o}^{\text {ideal }}$ is largely determined by $\Delta_{\text {int }}$; see Eqs. (48) and (55) and Fig. 7]. When the steps are located away from the center, it reduces $\Delta_{s}^{2 s s}\left(x_{s}\right)$ so that eventually at some point $\left|\Delta_{v o}^{\text {ideal }}\right|$ becomes larger than $\left|\Delta_{s}^{2 s s}\right|$. As such, we can expect that the valley splitting of a quantum dot with two symmetrically located steps is a also nonmonotonic function of the steps' location, $x_{s}$.

This behavior is clearly shown in the panel (a) of Fig. 11. The dash-dotted line of the figure shows the valley splitting obtained by neglecting $\Delta_{(\mathbf{1})}$, using Eqs. (48), (55) and (65), and setting $N_{0}=1$. The blue line is obtained by numerical calculation with all terms in Eq. (56) included. We observe that the effect of $\Delta_{(\mathbf{1})}$ is to further suppress the valley splitting as well as shift the step location where the valley splitting reaches its minimum. At this step location, $x_{s}=x_{m}^{2 s s} \sim 0.3 x_{0}$, the valley splitting is suppressed by more than $90 \%$. The panel (b) of the figure shows how the valley phase is changed as a function of the step location. As mentioned before, the sudden jump can be removed by using the $\pi$ periodicity of the valley phase.

We now study the electromagnetic dependence of the valley splitting for a quantum dot with two symmetrically locates interface steps. Given Fig. 11, away from $x_{m}^{2 s s}$, the dominant 

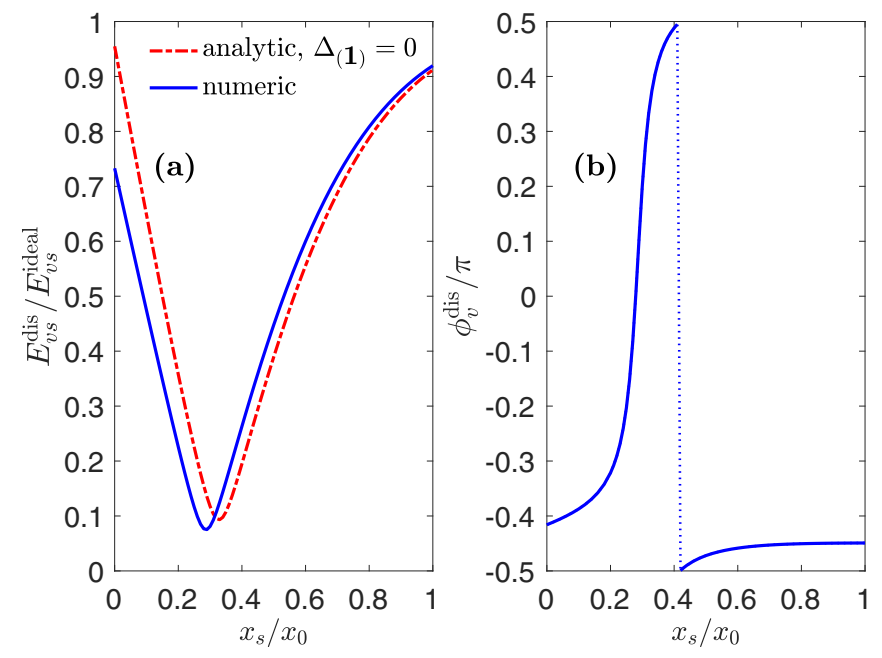

FIG. 11. (a) The normalized valley splitting for a quantum dot with two interface steps. (b) The valley phase as a function of the steps' location. In both panels, we used $B=0$ and $F_{z}=15 \mathrm{MV} / \mathrm{m}$.

contribution to the valley splitting is due to $\Delta_{v o}^{\text {ideal }}$ and $\Delta_{s}$. As such, we expect the scaling of the valley splitting with the electric field has to be approximately linear. This behavior is shown in the panel (a) of the Fig. 12. At the step locations close to $x_{m}^{2 s s}$, the contribution due to $\Delta_{(\mathbf{1})}$ becomes important so that we can expect a strong nonlinear dependency on the electric field; this is shown in the panel (b) of the figure. We
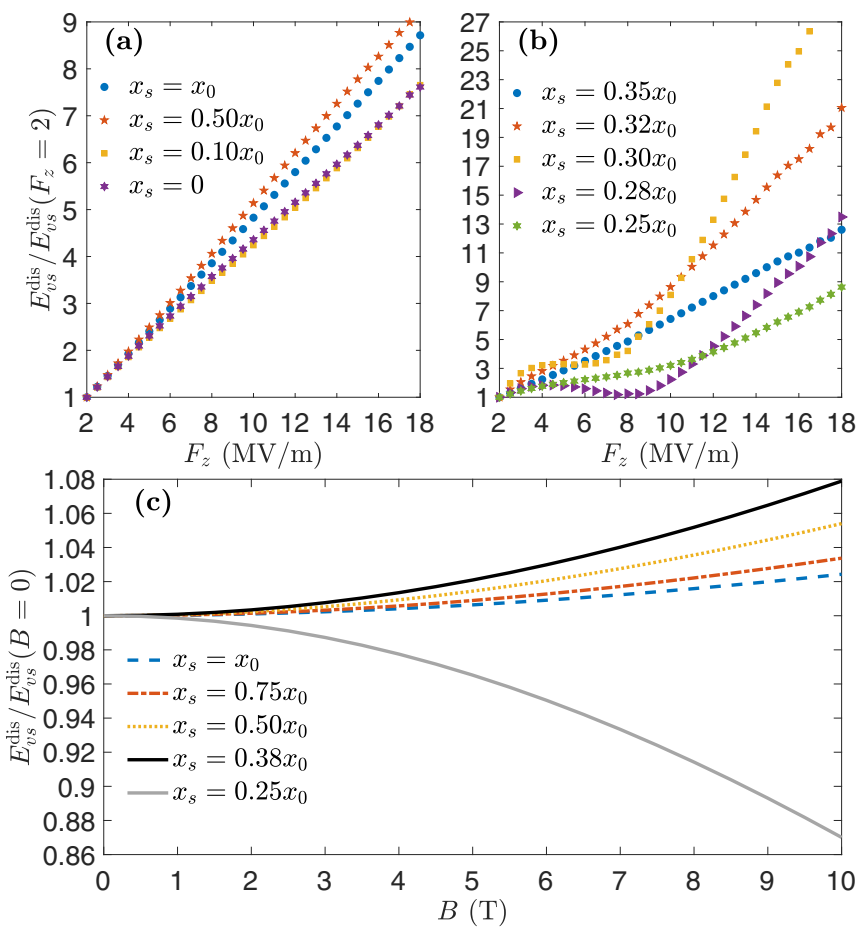

FIG. 12. [(a) and (b)] The normalized valley splitting as a function of the step location and electric field at $B=0$. (c) The normalized valley splitting as a function of an in-plane magnetic field. Similar to the Fig. 10(b), here we assumed $\mathbf{B}=$ $B(\cos \pi / 4, \sin \pi / 4,0)$ and $F_{z}=15 \mathrm{MV} / \mathrm{m}$.

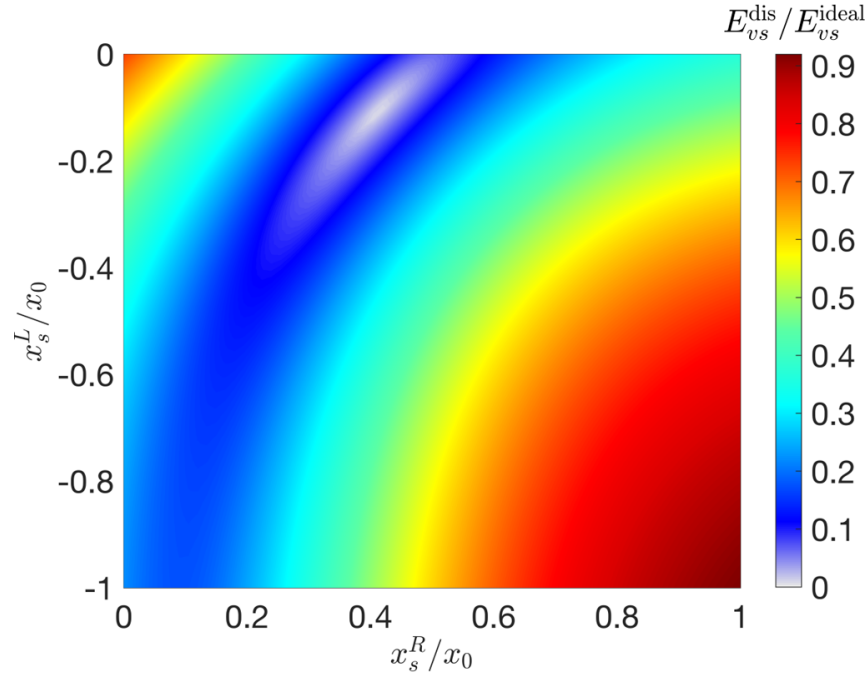

FIG. 13. The normalized valley splitting as a function of position of two interface steps at $B=0$ and $F_{z}=15 \mathrm{MV} / \mathrm{m}$.

note that such nonlinear scaling of the valley splitting with respect to the electric field is also reported in experiment [34].

In the panel (c) of the figure, we show how the valley splitting is changed by the magnetic field. For $x_{s}<x_{m}^{2 s s}$, the valley splitting is mainly controlled by $\Delta_{s}^{2 s s}$. As such, since this term reduces by the magnetic field, the valley splitting is also decreasing as well. For $x_{s}>x_{m}^{2 s s}, \Delta_{v o}^{\text {ideal }}$ is the dominant contribution to the valley splitting; therefore, the magnetic field always increases the valley splitting by reducing $\Delta_{s}^{2 s s}$ (and $\Delta_{(\mathbf{1})}$.) Note that this effect is stronger for the step locations where the slope of the curve shown in Fig. 11(a) is steeper.

Finally, we relax the condition of the two steps being symmetric around the quantum-dot center and use the step positions $x_{s 2}=x_{s}^{L} \leqslant 0$ and $x_{s 3}=x_{s}^{R} \geqslant 0$ in Eq. (32). In Fig. 13, we present our result for the normalized valley splitting as a function of the position of each step. Note that due to the nonsymmetric nature of the step potential, the valley splitting turns out not to be symmetric with respect to the location of the steps. We observe that the valley splitting can completely vanish in some specific configuration of the interface steps; this is also predicted in Ref. [22] using a simpler model. We have also studied the valley splitting for models including three and four interface steps, and we observed qualitatively similar behavior for the valley splitting as a function of electric and magnetic fields as presented in this section.

\section{SUMMARY AND CONCLUSIONS}

To summarize, the valley splitting is one of the important properties for the silicon quantum dots that directly influences the lifetime and scalability of silicon spin qubits. As such, understanding the behavior and tunability of the valley splitting is very important. In this work, we studied how the valley splitting responds to the electromagnetic field for both ideal and disordered quantum dots. We considered a realistic potential profile for a $\mathrm{SiGe} / \mathrm{Si} / \mathrm{SiGe}$ quantum dot by taking into account both lower and upper $\mathrm{Si} / \mathrm{SiGe}$ interfaces as well 
the interface between upper SiGe layer and the insulating layer hosting the gate electrodes; see Fig. 1 and Eq. (2). While so far the out-of-plane electron motion has been studied by variational methods in a simpler potential model including only one $\mathrm{Si} / \mathrm{SiGe}$ interface, we found the exact (within effective mass theory) envelope functions of the ground state as well as the excited states for the out-of-plane electron motion. This has enabled us to find the electron envelope function in the presence of finite magnetic field as well as interface disorder. In both cases, the envelope function reflects the coupling between in-plane to out-of-plane degrees of freedom, see Eqs. (25) and (33). Our analysis enables us to obtain the coupling coefficients using perturbation theory, for arbitrary configurations for the interface disorder.

We showed that in an ideal quantum dot, the valley splitting, within the leading order, always scales linearly with the out-of-plane electric field; see Fig. 7. Moreover, the valley splitting slightly increases with an applied in-plane magnetic field (by $2 \%$ for $B=10 \mathrm{~T}$ ) due to the coupling to the outof-plane excited states; see Fig. 8. The presence of interface disorder can significantly modify and suppress the valley splitting. We considered a stair-like disordered interface and studied the suppression of valley splitting due to the interface miscuts. We found up to $80 \%$ suppression caused by a single interface step, see Fig. 9, whereas two interface steps can completely diminish the valley splitting, see Fig. 13.

For a quantum dot with a disordered interface, we found that depending on the number and locations of the interface steps, the valley splitting can scale nonlinearly with the electric field; see the Fig. 10(a) and panels (a) and (b) of Fig. 12. If there is only one miscut at the interface, the magnetic field always increases the valley splitting (by up to $7 \%$ for $B=10 \mathrm{~T}$ ), see the Fig. 10(b). However, for multiple interface miscuts, the magnetic field can both increase or even suppress the valley splitting, depending on the configuration of the miscuts [we observed an increase by up to $8 \%$ and a decrease by up to $-13 \%$ for the set of parameters used in panel (c) of Fig. 12].

In the theory of spin relaxation induced by the valley coupling, one important set of quantities are the transition dipole matrix elements between the valley states $[3,18]$. For an ideal quantum dot, the envelope function has an in-plane mirror symmetry (Fig. 4). This, in turn, gives rise to vanishing of the in-plane dipole matrix elements. However, the presence of the interface disorder can break the in-plane mirror symmetry (Fig. 6). Our findings for the envelope function in the presence of disorder now enable the prediction of the dipole matrix elements as a function of the electromagnetic field. To our knowledge, the dipole matrix elements have always been taken as fitting parameters. Future work will be needed to further develop the theory of spin relaxation induced by the valley coupling based on a calculation of the transition dipole matrix elements and valley splitting in a magnetic field.

\section{ACKNOWLEDGMENTS}

We gratefully acknowledge useful discussions with $\mathrm{M}$. Russ and M. Benito. This work has been supported by ARO grant number W911NF-15-1-0149.

\section{APPENDIX A: BOUND STATES OF A TRIANGULAR POTENTIAL WELL: A SELF-CONSISTENT APPROXIMATION}

In this Appendix, we present our analysis leading to the ground-state energy and wavefunction given by Eqs. (14) and (15) for $\mathrm{SiGe} / \mathrm{Si} / \mathrm{SiGe}$ heterostructures. Furthermore, we argue that our analysis can be extended in order to include analytical expressions for the ground and first few excited states of $\mathrm{Si} / \mathrm{SiO}_{2}$ heterostructures. We start by making the reasonable assumption that in the case of SiGe barrier, the ground-state energy is much smaller than the interface potential $E_{z, 0} \ll U_{0}^{\mathrm{SiGe}}$. We now note that if the barrier is $\mathrm{SiO}_{2}$, the barrier potential is much higher, $U_{0}^{\mathrm{SiO}_{2}}=3 \mathrm{eV}$, than the SiGe barrier potential, $U_{0}^{\mathrm{SiGe}}=0.15 \mathrm{eV}$. Therefore, in this case, we can assume not only the ground-state energy but also the energy of the first few excited states as well are much smaller than the barrier potential, $E_{z, n} \ll U_{0}^{\mathrm{SiO}_{2}}$. This assumption enables us to neglect the thickness of the barrier (since in this case the eigenstate under the barrier quickly decays to zero). Moreover, as pointed out in Sec. II A, the electric field pushes the envelope function towards the upper barrier so that we can neglect the silicon layer thickness as well. Therefore we simplify the full potential [given by Eq. (2) for the $\mathrm{Si} / \mathrm{SiGe}$ quantum dot] and take it as $U(z)=U_{0}^{\text {barrier }} \theta(z)$.

Here we stress again that the following analysis is valid in the case of SiGe barrier only for $n=0$ (i.e., the ground state). For the excited states, the energy can become comparable to $U_{0}^{\mathrm{SiGe}}$ and therefore the thickness of the barrier has to be taken into account for a proper assessment. In such a case, one needs to rely on numerically solving Eq. (9). In the case of $\mathrm{SiO}_{2}$ barrier, $n$ is relaxed and contains both ground and excited states. We note that eventually for some high-lying excited states, the assumption of $E_{z, n} \ll U_{0}^{\mathrm{SiO}_{2}}$ breaks down. However, since those states are far split in energy from the ground state, their contribution in the perturbative analysis is negligible (see Table III). For simplicity, in the following we only use $U_{0}$ and bear in mind that the interface potential depends on the barrier.

We use the confinement length and energy given by Eqs. (4) and (5) to define the dimensionless quantity,

$$
\tilde{\zeta}_{n}(z)=\left\{\begin{array}{ll}
\tilde{U}_{0}-\tilde{z}-\tilde{\epsilon}_{z, n}, & z>0 \\
-\tilde{z}-\tilde{\epsilon}_{z, n} . & z \leqslant 0
\end{array} .\right.
$$

We then arrive to the below Schrödinger equation for the envelope function

$$
\frac{d^{2}}{d \tilde{z}^{2}} \psi_{z, n}-\tilde{\zeta}_{n} \psi_{z, n}=0
$$

Inside the silicon layer where $z \leqslant 0, \psi_{z, n}$ is given by the Airy function of the first kind,

$$
\psi_{z, n}=M_{n} z_{0}^{-1 / 2} \operatorname{Ai}\left(-\tilde{z}-\tilde{\epsilon}_{z, n}\right), \quad z \leqslant 0 .
$$

Let us now consider the form of the eigenstate inside the barrier; the exact solution for the envelope function, up to prefactors, reads $\operatorname{Ai}\left(\tilde{U}_{0}-\tilde{z}-\tilde{\epsilon}_{z, n}\right)$. However, in order to find an analytic relation for the energy, we try to approximate the envelope function in the barrier. If there was no electric field inside the barrier [i.e., the $-\tilde{z}$ term in $\tilde{\zeta}_{n}(z>0)$ was absent], the eigenstates would have been proportional to $\exp \left(-\sqrt{\tilde{U}_{0}-\tilde{\epsilon}_{z, n}} z\right)$. Due to the presence of the electric 
field, the potential barrier is reduced along $z$ and the wave function can further penetrate into the barrier. To take this into account, we introduce a parameter $\lambda_{n}$ into the exponent of the exponentially decaying wave function that allows further penetration into $\mathrm{SiGe}$ provided $\lambda_{n}<1$. We thus approximate the wave function inside the barrier by

$$
\psi_{z, n} \simeq M_{n} z_{0}^{\prime-1 / 2} \operatorname{Ai}\left(-\tilde{\epsilon}_{z, n}\right) e^{-\lambda \sqrt{\tilde{U}_{0}-\tilde{\epsilon}_{z, n} z}} . \quad z>0 .
$$

We now use the appropriate boundary condition concerning the first derivative of $\psi_{z, n}$ at the $z=0$ interface,

$$
\left.\frac{1}{m_{l}^{\mathrm{Si}}} \frac{d}{d z} \psi_{z, n}\right|_{z \rightarrow 0^{-}}=\left.\frac{1}{m_{l}^{\text {barrier }}} \frac{d}{d z} \psi_{z, n}\right|_{z \rightarrow 0^{+}} .
$$

Here, $m_{l}^{\mathrm{Si}}=0.98 m_{e}$ and $m_{l}^{\mathrm{SiGe}} \simeq m_{l}^{\mathrm{Si}}$. For the case of $\mathrm{Si} / \mathrm{SiO}_{2}$ quantum dot, we follow Ref. [37] and consider $\mathrm{SiO}_{2}$ as a direct gap crystalline material in which case $m_{l}^{\mathrm{SiGe}} \simeq 0.34 m_{e}$ (note that $\mathrm{SiO}_{2}$ can also be an amorphous material; in this case, the effective mass is not well defined, see Ref. [37] for details.) We then find the penetration parameter $\lambda_{n}$ from Eq. (A4),

$$
\lambda_{n}=\frac{m_{l}^{\text {barrier }}}{m_{l}^{\mathrm{Si}}} \frac{\operatorname{Ai}^{\prime}\left(-\tilde{\epsilon}_{z, n}\right)}{\operatorname{Ai}\left(-\tilde{\epsilon}_{z, n}\right)} \frac{1}{\sqrt{\tilde{U}_{0}-\tilde{\epsilon}_{z, n}}} .
$$

We now self-consistently determine the ground-state energy by noting,

$$
\tilde{\epsilon}_{z, n}=\frac{\left\langle\psi_{z, n}\left|\frac{d^{2}}{d \tilde{z}^{2}}-\tilde{z}+\tilde{U}_{0} \theta(\tilde{z})\right| \psi_{z, n}\right\rangle}{\left\langle\psi_{z, n} \mid \psi_{z, n}\right\rangle} .
$$

From the above equation and using Eqs. (A3) and (A5) we finally arrive at

$$
\begin{aligned}
&\left(\tilde{U}_{0}-\tilde{\epsilon}_{z, n}\right) \mathrm{Ai}^{\prime}\left(-\tilde{\epsilon}_{z, n}\right) \mathrm{Ai}^{2}\left(-\tilde{\epsilon}_{z, n}\right) \\
&=\frac{1}{2} \mathrm{Ai}^{3}\left(-\tilde{\epsilon}_{z, n}\right)+\operatorname{Ai}^{\prime 3}\left(-\tilde{\epsilon}_{z, n}\right) .
\end{aligned}
$$

In order to find the solution of the Eq. (A7), we note that for the infinite potential well $\tilde{U}_{0}=\infty$, the eigenenergy is determined by the roots of the Airy function, the first few of which read $-r_{0} \simeq-2.3381,-r_{1} \simeq-4.0879,-r_{2} \simeq$ $-5.5205,-r_{3} \simeq-6.7867$, and $-r_{4} \simeq-7.9441$. This suggests that so long as the eigenenergy is much smaller than the barrier potential, $\tilde{\epsilon}_{z, n} \ll \tilde{U}_{0}$, the solutions of Eq. (A7) should remain close to the roots of the Airy function. As such, we consider a solution of the form

$$
\tilde{\epsilon}_{z, n}=r_{n}+\delta \tilde{\epsilon}_{n},
$$

and expand $\mathrm{Ai}$ and $\mathrm{Ai}^{\prime}$ functions around $-r_{n}$. We keep terms up to quadratic order in $\delta \tilde{\epsilon}_{n}$ to find

$$
\begin{aligned}
& \operatorname{Ai}\left(-\tilde{\epsilon}_{z, n}\right)=-\delta \tilde{\epsilon}_{n} \operatorname{Ai}^{\prime}\left(-r_{n}\right)+\mathcal{O}\left(\delta \tilde{\epsilon}_{n}^{3}\right), \\
& \operatorname{Ai}^{\prime}\left(-\tilde{\epsilon}_{z, n}\right)=\operatorname{Ai}^{\prime}\left(-r_{n}\right)-\frac{1}{2} \delta \tilde{\epsilon}_{n}^{2} r_{n} \operatorname{Ai}^{\prime}\left(-r_{n}\right)+\mathcal{O}\left(\delta \tilde{\epsilon}_{n}^{3}\right) .
\end{aligned}
$$

By substituting Eqs. (A9) into Eq. (A7) and keeping terms up to quadratic order, we find $\tilde{U}_{0} \delta \tilde{\epsilon}_{n}^{2}=1$. This indicates that the leading order correction is independent on the energy level, $n$, and we find $\delta \tilde{\epsilon}= \pm 1 / \sqrt{\tilde{U}_{0}}$.

In order to determine which sign is physically acceptable, we note from Eqs. (A3b) and (A5) that the wave function decays inside the barrier provided $\mathrm{Ai}^{\prime}\left(-\tilde{\epsilon}_{z, n}\right) / \mathrm{Ai}\left(-\tilde{\epsilon}_{z, n}\right)>0$.
This is satisfied only if $\delta \tilde{\epsilon}$ has a negative sign. In this case, we also find $\lambda_{n}<1$, as expected. Note that if we have kept the expansions in Eq. (A9) up to the cubic order, we would have found a correction to the $\tilde{\epsilon}_{z, n}$ of order $\tilde{U}_{0}^{-3 / 2}$.

Finally, from the normalization of the envelope function, we find

$$
\begin{aligned}
M_{0}=\left[\tilde{\epsilon}_{z, 0} \operatorname{Ai}\left(-\tilde{\epsilon}_{z, 0}\right)^{2}+\operatorname{Ai}^{\prime}\left(-\tilde{\epsilon}_{z, 0}\right)^{2}\right. \\
\left.+\frac{1}{2} \frac{m_{l}^{\mathrm{Si}}}{m_{l}^{\text {barrier }}} \operatorname{Ai}\left(-\tilde{\epsilon}_{z, 0}\right)^{3} / \operatorname{Ai}^{\prime}\left(-\tilde{\epsilon}_{z, 0}\right)\right]^{-1 / 2} .
\end{aligned}
$$

In order to simplify this, we use Eqs. (A8) and (A9) and find $M_{n} \simeq 1 / \mathrm{Ai}^{\prime}\left(-r_{n}\right)$.

\section{APPENDIX B: ENERGY CORRECTION DUE TO THE MAGNETIC FIELD AND INTERFACE STEPS}

In Secs. II B and II C, we studied the corrections to the envelope function due to the presence of an in-plane magnetic field and interface steps. Here in this Appendix, we complement those sections by studying the energy correction to the ground-state energy up to the second order, $E_{0}^{\prime}=E_{0}^{(1)}+E_{0}^{(2)}$, for a $\mathrm{SiGe} / \mathrm{Si} / \mathrm{SiGe}$ quantum dot. In general, it follows from the standard stationary perturbation theory that the first- and second-order corrections to the ground-state energy read

$$
E_{0}^{(1)}=\left\langle\psi_{x, 0} \psi_{y, 0} \psi_{z, 0}\left|H_{\text {pert }}\right| \psi_{x, 0} \psi_{y, 0} \psi_{z, 0}\right\rangle
$$

and

$$
E_{0}^{(2)}=\sum_{m, q, n} \frac{\left|\left\langle\psi_{x, m} \psi_{y, q} \psi_{z, n}\left|H_{\text {pert }}\right| \psi_{x, 0} \psi_{y, 0} \psi_{z, 0}\right\rangle\right|^{2}}{-n \hbar \omega_{x}^{\prime}-q \hbar \omega_{y}^{\prime}+E_{z, 0}-E_{z, n}},
$$

where the in-plane confinement frequencies are given by Eqs. (21) and (22).

We start by considering the perturbation due to the presence of an in-plane magnetic field, in which case $H_{\text {pert }}$ is given by Eq. (18). We then immediately see that $E_{0}^{(1)}=0$, while we find for the second-order correction,

$$
\begin{aligned}
E_{0}^{(2)}= & -\frac{1}{4} \frac{m_{t}}{m_{l}} \hbar\left(\frac{\Omega_{B_{x}}^{2}}{\omega_{y}^{\prime}}+\frac{\Omega_{B_{y}}^{2}}{\omega_{x}^{\prime}}\right) \sum_{n=0}\left|\left\langle\psi_{z, n}|\partial / \partial \tilde{z}| \psi_{z, 0}\right\rangle\right|^{2} \\
& -\frac{1}{16} \sqrt{\frac{m_{t}}{m_{l}}} \frac{x_{0}^{\prime 2}}{l_{B_{x}}^{2}} \frac{y_{0}^{\prime 2}}{l_{B_{y}}^{2}} \frac{\hbar \Omega_{B_{x}} \Omega_{B_{y}}}{\omega_{x}^{\prime}+\omega_{y}^{\prime}} .
\end{aligned}
$$

Here, the cyclotron frequency $\Omega_{x(y)}$ and the magnetic length $l_{x(y)}$ are given by Eqs. (19) and (20). In Fig. 14, we use the set of parameters given by the caption of Table I (in particular $x_{0}=12 \mathrm{~nm}$ and $y_{0}=15 \mathrm{~nm}$ ) and show the correction to the ground-state energy relative to the (smaller) in-plane orbital splitting, $\left|E_{0}^{(2)}\right| / \hbar \omega_{y}^{\prime}$. We observe that even at $B=10 \mathrm{~T}$, this relative correction is nearly $2 \%$. We also note that the correction to the ground-state energy relative to the total unperturbed energy, $\left|E_{0}^{\prime}\right| / E_{0}$ where $E_{0}=(\hbar / 2)\left(\omega_{x}^{\prime}+\omega_{y}^{\prime}\right)+E_{z, 0}$, is about two orders of magnitude smaller than the values shown in the Fig. 14. This indicates that indeed applying the perturbation theory remains valid within all range of the considered magnetic fields.

We now move on to study the correction to the groundstate energy due to the presence of the interface steps. Here we 


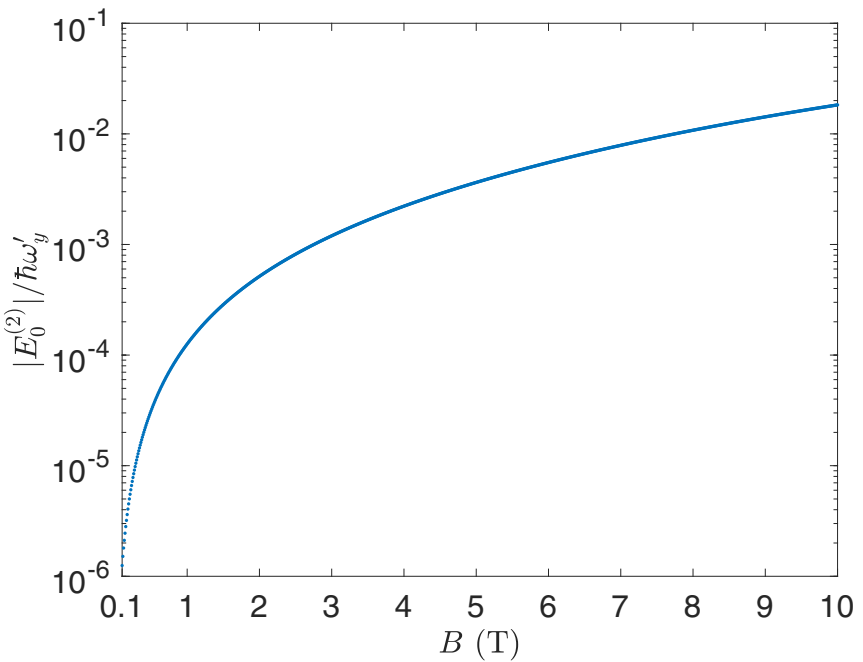

FIG. 14. The correction to the ground-state energy of an ideal quantum dot due to the presence of an in-plane magnetic field normalized to the in-plane orbital splitting. Similar to Fig. 8, here we have taken $\mathbf{B}=B(\cos (\theta), \sin (\theta), 0)$ and set $\theta=\pi / 4$.

consider a single interface step similar to what is considered in Sec. III C. We then find for the first-order correction,

$$
E_{0}^{(1)}=\frac{1}{4} \epsilon_{0} \operatorname{Erfc}\left(\sqrt{2} x_{s} / x_{0}^{\prime}\right) \frac{1-e^{\frac{a_{0}}{2 z_{0}}\left(\sqrt{\tilde{U}_{0}}-\frac{1}{2} \frac{1}{\sqrt{\bar{U}_{0}}}\right)}}{\sqrt{\tilde{U}_{0}}-\frac{1}{2} \frac{1}{\sqrt{\tilde{U}_{0}}}} .
$$

In order to calculate the second order correction, similar to Sec. III C, we only consider the largest contribution in Eq. (B2) corresponding to $m=1$ and $n=0$ (note that due to the symmetry of the interface steps along the $\hat{y}$ axis in our model, only the term with $q=0$ has a nonvanishing contribution.) We then find up to the leading order,

$$
E_{0}^{(2)} \simeq-\frac{2}{\pi} \frac{\left(E_{0}^{(1)}\right)^{2}}{\hbar \omega_{x}^{\prime}} \frac{e^{-4 x_{s}^{2} / x_{0}^{\prime 2}}}{\operatorname{Erfc}\left(\sqrt{2} x_{s} / x_{0}^{\prime}\right)^{2}}
$$

In Fig. 15, we use Eqs. (B4) and (B5) to show the first- and second-order corrections to the ground-state energy due to the presence of a single interface step at $x_{s}$. Here we also observe that the total correction to the energy is much smaller than the unperturbed ground-state energy (i.e., the ground-state energy of an ideal quantum dot.) Moreover, the second-order correction is much smaller than the first order correction. This is an indication that the perturbative analysis works properly. We have also calculated the correction to the ground-state energy in the presence of two interface steps, similar to our discussion in Sec. III C, and have found similar results as presented here. We therefore conclude in this Appendix that applying the perturbation theory is fully justified.

\section{APPENDIX C: SECOND-ORDER CORRECTION TO THE ENVELOPE FUNCTION DUE TO THE INTERFACE DISORDER}

Here we present the complete form of the second-order correction due to the interface steps. According to perturba-

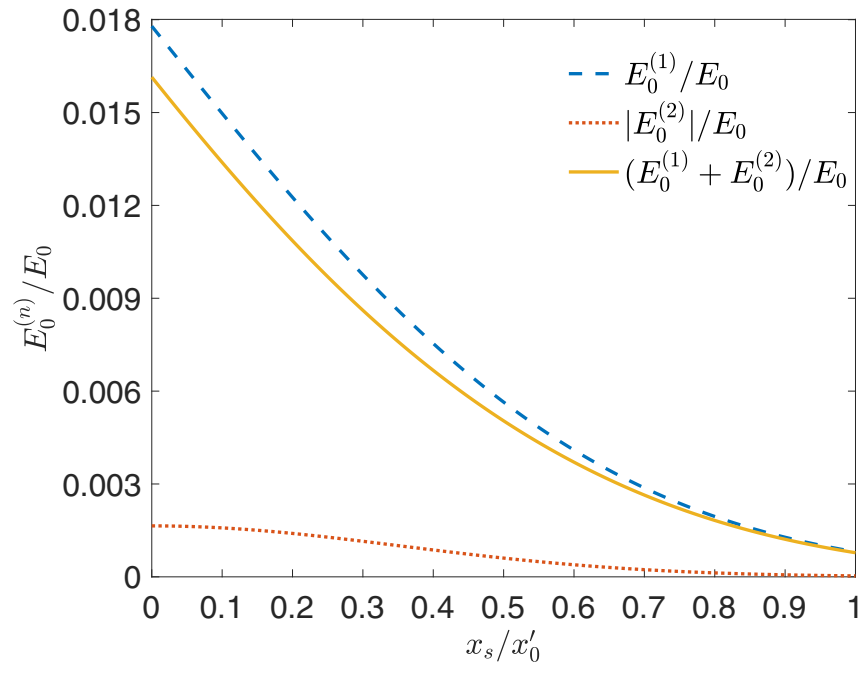

FIG. 15. The correction to the ground-state energy due to the presence of a single interface step at $x_{s}$ normalized to the unperturbed ground-state energy. Here we assumed $B=0$, while the other parameters are the same as given by Table I.

tion theory, we have

$$
\begin{aligned}
\mathcal{D}_{x y z, 0}^{(\mathbf{2})}= & \sum_{\left\{m^{\prime}, n^{\prime}\right\} \neq\{m, n\}} \sum_{\{m, n\} \neq\{0,0\}} \gamma_{m, n} \zeta_{m^{\prime}, n^{\prime}}^{m, n} \psi_{x, m^{\prime}} \psi_{z, n^{\prime}} \\
& -\sum_{\{m, n\} \neq\{0,0\}} \gamma_{m, n} c_{m, n} \psi_{x, m^{\prime}} \psi_{z, n^{\prime}},
\end{aligned}
$$

where $\gamma_{m, n}$ is given from Eq. (36) and here we defined

$$
\begin{aligned}
\zeta_{m^{\prime}, n^{\prime}}^{m, n} & =\frac{\left\langle\psi_{x, m^{\prime}} \psi_{z, n^{\prime}}\left|U_{\text {steps }}\right| \psi_{x, m} \psi_{z, n}\right\rangle}{E_{n, z}-E_{n^{\prime}, z}+\left(m-m^{\prime}\right) \hbar \omega_{x}^{\prime}}, \\
c_{m, n} & =\frac{\left\langle\psi_{x, 0} \psi_{z, 0}\left|U_{\text {steps }}\right| \psi_{x, 0} \psi_{z, 0}\right\rangle}{E_{0, z}-E_{n, z}-m \hbar \omega_{x}^{\prime}} .
\end{aligned}
$$

In order to arrive to Eq. (37), we only keep the dominant terms; i.e., in the set of $\gamma_{m, n}$, we keep $\gamma_{1,0}$, and in the set of $\zeta_{m^{\prime}, n^{\prime}}^{m, n}$, we keep $\zeta_{2,0}^{1,0}$ and $\zeta_{0,0}^{1,0}$. Finally, in the set of $c_{m, n}$, we keep $c_{1,0}$.

\section{APPENDIX D: VALLEY SPLITTING IN Si/SiO HETEROSTRUCTURES}

Here we build on our findings from Appendix A to study how the valley splitting in an ideal $\mathrm{Si} / \mathrm{SiO}_{2}$ heterostructure scale with the electric and magnetic fields. Following our discussion in Sec. III A and similar to Eq. (48), we can again separate the valley-orbit coupling into two terms accounting for the contribution due to the amplitude of the envelope function at the interface, $\Delta_{\text {int }}$, and another term containing the contribution due to the tail of the envelope function inside the $\mathrm{SiO}_{2}$ barrier, $\Delta_{t}$. Given Eqs. (A3a) and (49), the $\Delta_{\text {int }}$ contribution for a $\mathrm{Si} / \mathrm{SiO}_{2}$ heterostructure takes the same form as a $\mathrm{Si} / \mathrm{SiGe}$ heterostructure given by Eq. (51).

However, given Eqs. (A3b) and (50), we realize that due to the different effective masses between the $\mathrm{Si}$ (or $\mathrm{SiGe}$ ) and crystalline $\mathrm{SiO}_{2}$, the contribution due to the tail of the envelope function inside the barrier is changed from Eq. (52) 
TABLE III. The out-of-plane eigenenergies $E_{z, n}$ and coefficients $\alpha_{n}$ and $\beta_{n}$ (in units of inverse Tesla) for a $\mathrm{SiO}_{2}$ barrier at $F_{z}=$ $30 \mathrm{MV} / \mathrm{m}$. The other parameters are the same as given in the caption of Table I. Here the ground-state energy reads $E_{z, 0}=74.83 \mathrm{meV}$.

\begin{tabular}{cccc}
\hline \hline$n$ & $E_{z, n}(\mathrm{meV})$ & $\alpha_{n}\left(10^{-5} \mathrm{~T}^{-1}\right)$ & $\beta_{n}\left(10^{-5} \mathrm{~T}^{-1}\right)$ \\
\hline 1 & 133.50 & 1.84 & 1.84 \\
2 & 181.52 & 0.55 & 0.55 \\
3 & 223.97 & 0.28 & 0.28 \\
4 & 262.77 & 0.18 & 0.18 \\
5 & 298.93 & 0.12 & 1.12 \\
\hline \hline
\end{tabular}

and is modified to

$$
\Delta_{t}=-\Delta_{\mathrm{int}} \frac{m_{l}^{\mathrm{Si}}}{m_{l}^{\mathrm{SiO}_{2}}}\left[1-\frac{1}{2 \tilde{U}_{0}}+i \frac{k_{0} z_{0}}{\sqrt{\tilde{U}_{0}}}\right]^{-1} .
$$

Therefore, while in $\mathrm{Si} / \mathrm{SiGe}$ quantum dots, $\left|\Delta_{\text {int }}\right|$ is larger than $\left|\Delta_{t}\right|$ by nearly a factor of 6 (see Fig. 7), this ratio for $\mathrm{Si} / \mathrm{SiO}_{2}$ is reduced by $m_{l}^{\mathrm{SiO}_{2}} / m_{l}^{\mathrm{Si}}$ and becomes nearly a factor of 2 . This is also in agreement with the findings in Ref. [30] where a variational ansazt for the out-of-plane envelope function is employed. Given Eqs. (49) and (D1), we see that the dominant contribution to the valley-orbit coupling remains a linear function of electric field for an ideal $\mathrm{Si} / \mathrm{SiO}_{2}$ quantum dot [as the term $1 / \tilde{U}_{0} \propto F_{z}^{2 / 3}$ is only a negligible contribution in Eq. (D1)-also see Eq. (53)].

We now turn to study how the valley splitting of an ideal $\mathrm{Si} / \mathrm{SiO}_{2}$ quantum dot evolves with an in-plane magnetic field. We first note that the correction to the envelope function due to the presence of an in-plane magnetic field has the same form as given by Eq. (27), while the out-of-plane states $\psi_{z, n}$ and thus the perturbative coefficients $\alpha_{n}$ and $\beta_{n}$ are different compared with the $\mathrm{Si} / \mathrm{SiGe}$ quantum dots. As such, the evolution of the valley splitting in the magnetic field also remains the same as given by Eq. (55).

Here, in Table III, we show an example of the eigenenergies as well as the perturbative coefficients $\alpha_{n}$ and $\beta_{n}$. Note that the perturbative parameter $\eta$ from Eq. (30) is independent on the out-of-plane motion and is determined only from the in-plane confinement. We also note that for $\mathrm{Si} / \mathrm{SiO}_{2}$ heterostructures, the typical applied electric field is larger than the $\mathrm{Si} / \mathrm{SiGe}$ heterostructures and is reported to be $F_{z}=$ $30 \mathrm{MV} / \mathrm{m}$ in Ref. [3]. In this case, we find $z_{0} \simeq 1.12 \mathrm{~nm}$ and $\tilde{U}_{0} \simeq 89$. Due to this stronger electrical confinement, we observe in Table III that within the shown numerical precision, $\alpha_{n}=\beta_{n}$ while, noticeably, they take values that are nearly two orders of magnitude smaller compared with the values for $\mathrm{Si} / \mathrm{SiGe}$ (obtained at $F_{z}=15 \mathrm{MV} / \mathrm{m}$ ), see Table I. Moreover, the perturbative coefficients decay by one order of magnitude by taking up to five excited states enabling us to set $n_{\max }=5$ as the upper cutoff in the summation given by Eq. (27). We conclude here that the effect of an in-plane magnetic field to the valley splitting of an ideal $\mathrm{Si} / \mathrm{SiO}_{2}$ is generally much smaller than that of a $\mathrm{Si} / \mathrm{SiGe}$ quantum dot (due to significantly smaller $\alpha_{n}$ and $\beta_{n}$ coefficients).

Let us now consider a single step at the $\mathrm{Si} / \mathrm{SiO}_{2}$ interface; we note from Eqs. (60) and (61) that since the interface potential $U_{0}$ is much larger compared to $\mathrm{Si} / \mathrm{SiGe}$ quantum dots, the adverse effect of $\Delta_{s}^{1 s}$ and $\Delta_{1}^{1 s}$ becomes smaller. In particular, we find at $x_{s}=0$ the valley splitting is reduced by only around $50 \%$ (as opposed to nearly $80 \%$ suppression at $x_{s}=0$ for $\mathrm{Si} / \mathrm{SiGe}$, see Fig. 9). We would like to add here that disorder has a more complex nature in $\mathrm{Si} / \mathrm{SiO}_{2}$ heterostructures compared with the $\mathrm{Si} / \mathrm{SiGe}$ heterostructures. While in the latter case, the disorder happens through atomic steps at the interafce (i.e. height of the interface potential), in $\mathrm{Si} / \mathrm{SiO}_{2}$ quantum dots, in addition to the interface atomic steps, there can be disorder through a missing Oxygen atom. In Ref. [37], it has been shown that such a disorder can actually increase the valley splitting.

\section{APPENDIX E: VALLEY SPLITTING OF A DISORDERED QUANTUM DOT IN MAGNETIC FIELD: HIGHER-ORDER TERMS}

In this Appendix, we present the form of the sub-leading contribution $\Delta_{(2)}$ in Eq. (56). Using Eqs. (31) and (33), we find

$$
\begin{aligned}
\Delta^{(\mathbf{2})}(\mathbf{B})=\mathcal{C}_{0} & \left\{B_{x}^{2}\left(\frac{y_{0}^{\prime}}{z_{0}}\right)^{2} \sum_{n, n^{\prime}} \alpha_{n} \alpha_{n^{\prime}} \int_{-\infty}^{\infty} e^{-2 i k_{0} z} \psi_{x, 0}\left(B_{y}\right)^{2} \psi_{z, n} \psi_{z, n^{\prime}} U_{\text {steps }}(x, z) d x d z\right. \\
& +B_{y}^{2}\left(\frac{x_{0}^{\prime}}{z_{0}}\right)^{2} \sum_{n, n^{\prime}} \beta_{n} \beta_{n^{\prime}} \int_{-\infty}^{\infty} e^{-2 i k_{0} z} \psi_{x, 1}\left(B_{y}\right)^{2} \psi_{z, n} \psi_{z, n^{\prime}} U_{\text {steps }}(x, z) d x d z \\
& +B_{x}^{2} B_{y}^{2} x_{0}^{\prime 2} y_{0}^{\prime 2} \eta^{2} \int_{-\infty}^{\infty} e^{-2 i k_{0} z} \psi_{x, 1}\left(B_{y}\right)^{2} \psi_{z, 0}^{2} U_{\text {steps }}(x, z) d x d \\
& +\sum_{m, n, n^{\prime}} \gamma_{m, n} \gamma_{m, n^{\prime}} \int_{-\infty}^{\infty} U(z) e^{-2 i k_{0} z} \psi_{z, n} \psi_{z, n^{\prime}} d z \\
& \left.+\sum_{m, n, m^{\prime}, n^{\prime}} \gamma_{m, n} \gamma_{m^{\prime}, n^{\prime}} \int_{-\infty}^{\infty} e^{-2 i k_{0} z} \psi_{z, n} \psi_{z, n^{\prime}} \psi_{x, m}\left(B_{y}\right) \psi_{x, m^{\prime}}\left(B_{y}\right) U_{\text {steps }}(x, z) d x d z\right\} .
\end{aligned}
$$

The perturbative coefficients $\alpha, \beta, \eta$ and $\gamma$ are given in Secs. II B and II C, and using the excited states $\psi_{z, n}$ from Sec. II A, we can numerically evaluate the integrals. 


\section{APPENDIX F: THE EFFECT OF OUT-OF-PLANE EXCITED STATES IN $\Delta_{(1)}$}

In this Appendix, we explain in more detail the effect of the excited states $\psi_{z, n \geqslant 1}$ to the valley splitting for a quantum dot with single interface step. The step potential Eq. (32) in this case becomes

$$
U_{\text {steps }}^{1 s}(x, z)=-U_{0} \theta\left(x-x_{s}\right) \theta(z) \theta\left(z-\frac{a_{0}}{4}\right) .
$$

In Sec. III C, we found the contributions from $\Delta_{s}^{1 s}$ and $\Delta_{(\mathbf{1}),\{1,0\}}^{1 s}$ are out-of-phase with $\Delta_{v o}^{\text {ideal }}$, therefore, these terms would monotonically suppress the valley splitting. Here we show that once the out-of-plane excited states $\psi_{z, n}$ are taken into account, the real part of $\Delta_{(\mathbf{1})}^{1 s}$ gives rise to a nonmonotonic behavior of the valley splitting as a function of the step location, as shown in Fig. 9(a).

To see this effect, let us integrate Eq. (59) over $z$ by parts to find,

$$
\begin{aligned}
f_{m, n} \simeq & i U_{0} \frac{1}{2 k_{0}} \int_{x_{s}}^{\infty} \psi_{x, m} \psi_{x, 0} d x \\
& \times\left[\psi_{z, n}(0) \psi_{z, 0}(0)-e^{-i k_{0} a_{0} / 2} \psi_{z, n}\left(\frac{a_{0}}{4}\right) \psi_{z, 0}\left(\frac{a_{0}}{4}\right)\right],
\end{aligned}
$$

where we have neglected the small contributions containing integral over $e^{-2 i k_{0} z} \psi_{z, n} \psi_{z, 0}^{\prime}$ and $e^{-2 i k_{0} z} \psi_{z, n}^{\prime} \psi_{z, 0}$. As we have shown in Sec. III A, the dominant contribution in $\Delta_{v o}^{\text {ideal }}$ is given by $\Delta_{\text {int }}$ from Eq. (51) which is an imaginary quantity and is due to the amplitude of the envelope function at the $\mathrm{Si} / \mathrm{SiGe}$ interface.

Given Eqs. (F2) and (58), the imaginary part of $\Delta_{(\mathbf{1})}^{1 s}$ is in opposite phase with $\Delta_{\text {int }}$ [note that in Eq. (F2) we have

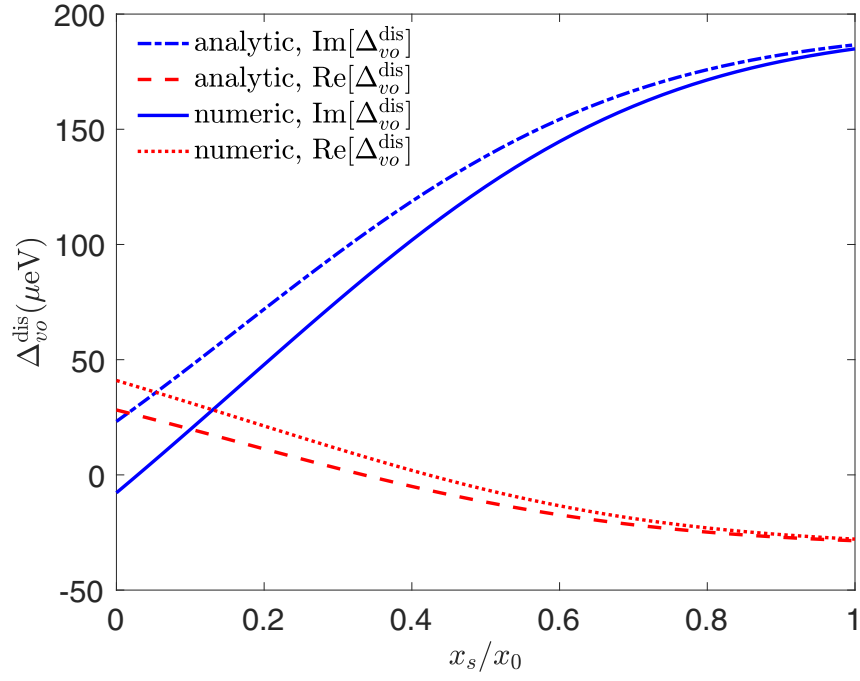

FIG. 16. Imaginary and real parts of the valley-orbit coupling for a quantum dot with single interface step at $\mathbf{B}=0$ and $F_{z}=15$ $\mathrm{MV} / \mathrm{m}$. In order to obtain the analytical results, we used $\Delta_{v o}^{\text {ideal }}$ from Eq. (48), $\Delta_{s}^{1 s}$ from Eq. (60) and $\Delta_{(\mathbf{1}),\{1,0\}}^{1 s}$ from Eq. (61). For the numerical results, we calculated all contributions in Eq. (56) including terms with $n \geqslant 1$ in $\Delta_{(\mathbf{1}),\{m, n\}}^{1 s}$.

$\left.e^{-i k_{0} a_{0} / 2}=-0.891-0.454 i\right]$. Therefore the closer the step is located to the quantum dot center, the more the imaginary part of total valley-orbit coupling is suppressed. On the other hand, the real part of the valley-orbit coupling is increased when the step is closer to the center, and at some point it becomes the dominant contribution to the total valley-orbit coupling. We plot the imaginary and real parts of the valley orbit coupling in Fig. 16. The lines labeled analytic are obtained by using the analytic relations we obtained in Sec. III C for $D_{s}^{1 s}$ and $D_{(\mathbf{1}),\{1,0\}}^{1 s}$ whereas the lines labeled numeric are found from numerically evaluating the valley-orbit coupling including all terms in $\Delta_{(\mathbf{1}),\{m, n\}}^{1 s}$. We observe that the nonmonotonic behavior of the valley splitting as a function of step location can be seen only by taking into account the out-of-plane excited states $\psi_{z, n}$.
[1] F. A. Zwanenburg, A. S. Dzurak, A. Morello, M. Y. Simmons, L. C. L. Hollenberg, G. Klimeck, S. Rogge, S. N. Coppersmith, and M. A. Eriksson, Rev. Mod. Phys. 85, 961 (2013).

[2] A. Morello, J. J. Pla, F. A. Zwanenburg, K. W. Chan, K. Y. Tan, H. Huebl, M. Möttönen, C. D. Nugroho, C. Yang, J. A. van Donkelaar, A. D. C. Alves, D. N. Jamieson, C. C. Escott, L. C. L. Hollenberg, R. G. Clark, and A. S. Dzurak, Nature (London) 467, 687 (2010).

[3] C. H. Yang, A. Rossi, R. Ruskov, N. S. Lai, F. A. Mohiyaddin, S. Lee, C. Tahan, G. Klimeck, A. Morello, and A. S. Dzurak, Nat. Commun. 4, 2069 (2013).

[4] F. Borjans, D. M. Zajac, T. M. Hazard, and J. R. Petta, Phys. Rev. Applied 11, 044063 (2019).

[5] L. V. C. Assali, H. M. Petrilli, R. B. Capaz, B. Koiller, X. Hu, and S. Das Sarma, Phys. Rev. B 83, 165301 (2011).
[6] A. M. Tyryshkin, S. Tojo, J. J. L. Morton, H. Riemann, N. V. Abrosimov, P. Becker, H.-J. Pohl, T. Schenkel, M. L. W. Thewalt, K. M. Itoh, and S. A. Lyon, Nat. Mater. 11, 143 (2012)

[7] M. Steger, K. Saeedi, M. L. W. Thewalt, J. J. L. Morton, H. Riemann, N. V. Abrosimov, P. Becker, and H.-J. Pohl, Science 336, 1280 (2012).

[8] X. Mi, M. Benito, S. Putz, D. M. Zajac, J. M. Taylor, G. Burkard, and J. R. Petta, Nature (London) 555, 599 (2018).

[9] N. Samkharadze, G. Zheng, N. Kalhor, D. Brousse, A. Sammak, U. C. Mendes, A. Blais, G. Scappucci, and L. M. K. Vandersypen, Science 359, 1123 (2018).

[10] M. Veldhorst, J. C. C. Hwang, C. H. Yang, A. W. Leenstra, B. de Ronde, J. P. Dehollain, J. T. Muhonen, F. E. Hudson, K. M. Itoh, A. Morello, and A. S. Dzurak, Nat. Nanotechnol. 9, 981 (2014). 
[11] J. Yoneda, K. Takeda, T. Otsuka, T. Nakajima, M. R. Delbecq, G. Allison, T. Honda, T. Kodera, S. Oda, Y. Hoshi, N. Usami, K. M. Itoh, and S. Tarucha, Nat. Nanotechnol. 13, 102 (2018).

[12] D. M. Zajac, A. J. Sigillito, M. Russ, F. Borjans, J. M. Taylor, G. Burkard, and J. R. Petta, Science 359, 439-442 (2018).

[13] T. F. Watson, S. G. J. Philips, E. Kawakami, D. R. Ward, P. Scarlino, M. Veldhorst, D. E. Savage, M. G. Lagally, M. Friesen, S. N. Coppersmith, M. A. Eriksson, and L. M. K. Vandersypen, Nature (London) 555, 633 (2018).

[14] W. Huang, C. H. Yang, K. W. Chan, T. Tanttu, B. Hensen, R. C. C. Leon, M. A. Fogarty, J. C. C. Hwang, F. E. Hudson, K. M. Itoh, A. Morello, A. Laucht, and A. S. Dzurak, Nature (London) 569, 532 (2019).

[15] L. E. Golub and E. L. Ivchenko, Phys. Rev. B 69, 115333 (2004).

[16] M. Veldhorst, R. Ruskov, C. H. Yang, J. C. C. Hwang, F. E. Hudson, M. E. Flatté, C. Tahan, K. M. Itoh, A. Morello, and A. S. Dzurak, Phys. Rev. B 92, 201401(R) (2015).

[17] R. Ferdous, E. Kawakami, P. Scarlino, M. P. Nowak, D. R. Ward, D. E. Savage, M. G. Lagally, S. N. Coppersmith, M. Friesen, M. A. Eriksson, L. M. K. Vandersypen, and R. Rahman, npj Quantum Inf 4, 26 (2018).

[18] P. Huang and X. Hu, Phys. Rev. B 90, 235315 (2014).

[19] M. Friesen, S. Chutia, C. Tahan, and S. N. Coppersmith, Phys. Rev. B 75, 115318 (2007).

[20] A. L. Saraiva, M. J. Calderón, Rodrigo B. Capaz, Xuedong Hu, S. Das Sarma, and B. Koiller, Phys. Rev. B 84, 155320 (2011).

[21] Y. Wu and D. Culcer, Phys. Rev. B 86, 035321 (2012).

[22] B. Tariq and X. Hu, Phys. Rev. B 100, 125309 (2019).

[23] R. Ruskov, M. Veldhorst, A. S. Dzurak, and C. Tahan, Phys. Rev. B 98, 245424 (2018).

[24] R. M. Jock, N. T. Jacobson, P. Harvey-Collard et al., Nat. Commun. 9, 1768 (2018).

[25] H. J. W. Zandvliet and H. B. Elswijk, Phys. Rev. B 48, 14269 (1993).
[26] A. Hollmann, T. Struck, V. Langrock, A. Schmidbauer, F. Schauer, K. Sawano, H. Riemann, N. V. Abrosimov, D. Bougeard, and L. R. Schreiber, Phys. Rev. Applied 13, 034068 (2020).

[27] M. Friesen, M. A. Eriksson, and S. N. Coppersmith, Appl. Phys. Lett. 89, 202106 (2006).

[28] S. Goswami, K. A. Slinker, M. Friesen, L. M. McGuire, J. L. Truitt, C. Tahan, L. J. Klein, J. O. Chu, P. M. Mooney, D. W. van der Weide, R. Joynt, S. N. Coppersmith, and M. A. Eriksson, Nat. Phys. 3, 41 (2007).

[29] See, for instance, J. H. Davies, The physics of Low-Dimensional Semiconductors (Cambridge Univercity Press, 1998).

[30] A. L. Saraiva, M. J. Calderón, X. Hu, S. Das Sarma, and B. Koiller, Phys. Rev. B 80, 081305(R) (2009).

[31] X. Mi, J. V. Cady, D. M. Zajac, J. Stehlik, L. F. Edge, and J. R. Petta, Appl. Phys. Lett. 110, 043502 (2017).

[32] Virginia Semiconductors, The General Properties of Si, Ge, $\mathrm{SiGe}, \mathrm{SiO}_{2}$ and $\mathrm{Si}_{3} \mathrm{~N}_{4}$, available at: https://www.virginiasemi. com/pdf/generalpropertiesSi62002.pdf.

[33] T. B. Boykin, G. Klimeck, M. A. Eriksson, M. Friesen, S. N Coppersmith, P. von Allmen, F. Oyafuso, and S. Lee, Appl. Phys. Lett. 84, 115 (2004).

[34] S. F. Neyens, R. H. Foote, B. Thorgrimsson, T. J. Knapp, T. McJunkin, L. M. K. Vandersypen, P. Amin, N. K. Thomas, J. S. Clarke, D. E. Savage, M. G. Lagally, Mark Friesen, S. N. Coppersmith, and M. A. Eriksson, Appl. Phys. Lett. 112, 243107 (2018).

[35] B. P. Wuetz, M. P. Losert, A. Tosato, M. Lodari, P. L. Bavdaz, L. Stehouwer, P. Amin, J. S. Clarke, S. N. Coppersmith, A. Sammak, M. Veldhorst, M. Friesen, and G. Scappucci, arXiv:2006.02305.

[36] P. Boross, G. Széchenyi, D. Culcer, and A. Pályi, Phys. Rev. B 94, 035438 (2016)

[37] A. L. Saraiva, B. Koiller, and M. Friesen, Phys. Rev. B 82, 245314 (2010). 